\title{
Computing the Homology of Semialgebraic Sets. I: Lax Formulas*
}

\author{
Peter Bürgisser ${ }^{\dagger}$ \\ Technische Universität Berlin \\ Institut für Mathematik \\ GERMANY \\ pbuerg@math.tu-berlin.de
}

\author{
Felipe Cucker $\ddagger$ \\ Dept. of Mathematics \\ City University of Hong Kong \\ HONG KONG \\ macucker@cityu.edu.hk
}

\author{
Josué Tonelli-Cueto \\ Technische Universität Berlin \\ Institut für Mathematik \\ GERMANY \\ ton-cue@math.tu-berlin.de
}

\begin{abstract}
We describe and analyze an algorithm for computing the homology (Betti numbers and torsion coefficients) of closed semialgebraic sets given by Boolean formulas without negations over lax polynomial inequalities. The algorithm works in weak exponential time. This means that outside a subset of data having exponentially small measure, the cost of the algorithm is single exponential in the size of the data.

All previous algorithms solving this problem have doubly exponential complexity (and this is so for almost all input data). Our algorithm thus represents an exponential acceleration over state-of-the-art algorithms for all input data outside a set that vanishes exponentially fast.
\end{abstract}

AMS classification numbers: 14P10, 65D18, 65Y20, 68Q25

Keywords: Homology groups, Weak complexity, Numerical algorithms

\section{Introduction}

A semialgebraic set is a subset of $\mathbb{R}^{n}$ defined by a Boolean combination (i.e., a sequence of unions, intersections, and complements) of polynomial equalities and inequalities. The class of such sets is closed under unions, intersections, complements, and projections as well as under taking images and preimages of polynomial maps. This wealth of closure properties is consistent with the wealth of shapes that semialgebraic sets can take.

Semialgebraic sets play a distinguished role in several branches of mathematics. In mathematical logic, they occur as the definable sets of the first-order theory of real closed fields [62], in real algebraic geometry, where they are the constructible sets [7, 9], in

\footnotetext{
${ }^{*}$ This work was supported by the Einstein Foundation, Berlin.

${ }^{\dagger}$ Partially funded by DFG research grant BU 1371/2-2.

${ }^{\ddagger}$ Partially supported by a GRF grant from the Research Grants Council of the Hong Kong SAR (project number CityU 11302418).
} 
complexity theory, where complete problems over the reals in various complexity classes are stated in terms of semialgebraic sets [8, 12], in mathematical programming [10], robotics $[17,53], \ldots$ Not surprisingly, in the last decades, a substantial amount of work was devoted to the design of algorithms for problems involving semialgebraic sets. This goal was already present in Tarski's work [62], where a procedure to decide the first-order theory of the reals is given. In the 1970's Collins [23] and Wüthrich [65], independently introduced an algorithm, today known as Cylindrical Algebraic Decomposition (and usually referred as CAD) that allowed to solve most of the problems mentioned before. The cost of running CAD on a list of $q$ polynomials of degree at most $D$ in $n$ variables is $(q D)^{2^{\mathcal{O}(n)}}$, that is, it has a doubly exponential dependence on the number of variables, and this dependence is generic: it does hold for all choices of coefficients for the polynomials in the list outside a smaller dimensional set. In addition, since in all applications one needs to first compute a CAD and only then solve from this CAD the problem at hand, this generic doubly exponential complexity appears to be unavoidable no matter the problem considered. A new set of ideas, known as the critical points method, was proposed in the late 1980's by Grigori'ev and Vorobjov [38, 37]. Using these ideas, complexity bounds improved to singly exponential in $n,(q D)^{\mathcal{O}(n)}$, for many of the questions considered on semialgebraic sets: deciding emptiness [38, 49, 4], counting connected components [5, 18, 19, 39, 41], computing the dimension [43], the Euler-Poincaré characteristic [2], and the first few [3] Betti numbers.

A problem that was conspicuously left out of these improvements is that of computing the sequence of homology groups of a semialgebraic set. Of course, the list above contains partial results in this direction (the number of connected components being the 0th Betti number) but, as of today, no single exponential algorithm has been devised returning the whole sequence of homology groups over $\mathbb{Z}$ (which gives information about the torsion in addition to the Betti numbers). This sequence being arguably the most important set of topological invariants, the importance of its computation can hardly be overemphasized.

All the algorithms mentioned above are symbolic in the sense that they assume infinite precision in the computations. If implemented with finite precision they may, and experience shows they often do, return meaningless outputs. Driven by a search of numerical stability, Cucker and Smale devised a numerical algorithm for deciding emptiness [30]. The possibility of round-off errors implies the existence of a set of inputs for which, no matter the machine precision nor the algorithm at hand, there exists computations that return a wrong answer (wrong number of connected components, wrong dimension, etc.). This set of inputs, referred to as ill-posed, is usually lower dimensional and hence of measure zero in the space of data. Numerical algorithms are not expected to return an output on them. Instead, the computation time is expected to increase with the proximity to the set of ill-posed inputs, a proximity which is usually measured by, or closely related to, the condition number of the input at hand. Complexity estimates are therefore commonly expressed in the dimensions of the data and the condition number.

Yet, condition numbers are not, in general, known a priori, a fact that makes conditionbased complexity bounds to be less informative. The standard way to overcome this drawback, pioneered by Goldstine and von Neumann [36], Demmel [31], and Smale [59] among others, consists of accepting statistical bounds in exchange of eliminating the condition number from these bounds. To do so, the space of data is endowed with a probability measure (usually a Gaussian on an Euclidean space or the uniform distribution on a sphere) that allows one to treat the condition number as a random variable. In this setting, the most common form of analysis is the average analysis, that aims to bound the expected 
running time of the algorithm in terms of the data's dimensions only. Recently, however, Amelunxen and Lotz [1] brought in a different form of analysis with the aim of giving a theoretical explanation of the efficiency in practice of numerical algorithms whose average complexity was too high. A paradigm of this situation is the power method to compute the leading eigenpair of a Hermitian matrix: this algorithm is very fast in practice, yet the average number of iterations it performs has been shown to be infinite [44]. Amelunxen and Lotz realized that here, as in many other problems, this disagreement between theory and practice is due to the presence of a vanishingly small (more precisely, having a measure exponentially small with respect to the input size) set of outliers, outside of which the algorithm can be shown to be efficient. Complexity estimates holding outside a set of exponentially small measure were called weak in [1].

The lines above are the background against which we can state our main result.

Statement of the main result. Let $n \geq 2, q \geq 1$, and $\boldsymbol{d}=\left(d_{1}, \ldots, d_{q}\right)$, with $d_{i} \geq 1$ for $i=1, \ldots, q$. We denote by $\mathcal{P}_{\boldsymbol{d}}[q]$ the vector space of polynomial tuples $p=\left(p_{1}, \ldots, p_{q}\right)$ with $p_{i} \in \mathbb{R}\left[X_{1}, \ldots, X_{n}\right]$ of degree at most $d_{i}$. We let $D:=\max \left\{d_{1}, \ldots, d_{q}\right\}$ and denote by $N$ the dimension of $\mathcal{P}_{\boldsymbol{d}}[q]$. We endow this space with the Weyl inner product (see $\S 3.1$ below) and the resulting unit sphere $\mathbb{S}\left(\mathcal{P}_{\boldsymbol{d}}[q]\right)=\mathbb{S}^{N-1}$ with the uniform probability measure.

We say that a Boolean combination $\Phi$ over $p \in \mathcal{P}_{\boldsymbol{d}}[q]$ is lax if it has no complements; only unions and intersections of the atomic sets $\left\{p_{i}(x) \leq 0\right\},\left\{p_{i}(x)=0\right\}$, and $\left\{p_{i}(x) \geq 0\right\}$, for $i=1, \ldots, q$ (see $\S 2.1$ ). The size of $\Phi$, denoted size $(\Phi)$ is the number of unions and intersections in the sequence. We note that $\Phi$ defines a closed semialgebraic subset of $\mathbb{R}^{n}$ which we will denote by $W(p, \Phi)$. Finally, we associate to $p$ a condition number $\bar{\kappa}_{\text {aff }}(p)$ (whose precise definition is in $\S 7.1$ ). This condition number does not depend on $\Phi$.

The size of a pair $(p, \Phi)$ is $\operatorname{size}(p, \Phi):=N+\operatorname{size}(\Phi)$.

Theorem 1.1. We exhibit a stable numerical algorithm Homology that, given a tuple $p \in$ $\mathcal{P}_{\boldsymbol{d}}[q]$ and a lax Boolean combination $\Phi$ over $p$, computes the homology groups of $W(p, \Phi)$. The cost of Homology on input $(p, \Phi)$, denoted cost $(p, \Phi)$, that is, the number of arithmetic operations and comparisons in $\mathbb{R}$, satisfies:

(i) $\operatorname{cost}(p, \Phi) \leq \operatorname{size}(\Phi) q^{\mathcal{O}(n)}\left(n D \bar{\kappa}_{\text {aff }}(p)\right)^{\mathcal{O}\left(n^{2}\right)}$.

Furthermore, if $p$ is drawn from the uniform distribution on $\mathbb{S}^{N-1}$, then:

(ii) $\operatorname{cost}(p, \Phi) \leq \operatorname{size}(\Phi) q^{\mathcal{O}(n)}(n D)^{\mathcal{O}\left(n^{3}\right)}$ with probability at least $1-(n q D)^{-n}$, and

(iii) $\operatorname{cost}(p, \Phi) \leq 2^{\mathcal{O}\left(\operatorname{size}(p, \Phi)^{1+\frac{2}{D}}\right)}$ with probability at least $1-2^{-\operatorname{size}(p, \Phi)}$.

Observation 1.1.1. A few comments on Theorem 1.1 are called for.

(i) A detailed explanation, along with a proof, of the numerical stability mentioned in the statement above is in Section 7 of [29]. As the same explanation, word by word, applies in our context we will not deal with this issue in the rest of our exposition.

(ii) Part (iii) of Theorem 1.1 shows, in the terminology introduced by [1], that Homology works in weak exponential time.

(iii) It is easy to check that all the routines in algorithm Homology do parallelize. The parallel version of the algorithm can then be shown to work in parallel time $\operatorname{size}(p, \Phi)^{\mathcal{O}(1)}$ with probability at least $1-2^{-\operatorname{size}(p, \Phi)}$. That is, it works in weak parallel polynomial time. We will be more precise in $\S 7.4$. 
Relations with previous work and new ideas. Our results have not grown in the vacuum. They owe ideas and intuitions to a number of works in the literature. Our use of grids goes back to [30]. The goal in that paper was deciding feasibility of semialgebraic systems. Subsequently, these ideas were extended to the problem of counting the solutions of 0 -dimensional real projective sets [26, 27, 28] and, much more recently, to the computation of homology groups. In [29], it is the homology of real projective sets, and in [15], that of basic semialgebraic sets. Some of the objects in these two papers - notably the algebraic neighborhoods of a semialgebraic set given by $f=0, g \geq 0$ that were introduced in the last one (see $\S 2.5$ below) - play a crucial role in our development. Yet, the road-map to compute the homology groups of the closed set $W$ at hand passes, in both papers, through computing a covering $\mathcal{U}$ of $W$ by open balls of the same radius such that the nearest-point map $\mathcal{U} \rightarrow W$ induces a deformation retraction. When $W$ is a general closed semialgebraic set, however, such a covering may well not exist as the nearest point may be undefined at points arbitrarily close to $W$. A simple example of such a set $W$ is given in the following picture.

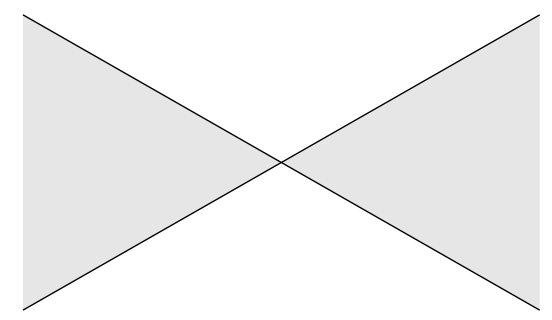

We therefore need to proceed differently (see Remark 2.5 below for more on this need). Our idea is to aim for a covering $\mathcal{U}$ which is only homologically equivalent to $S$. To obtain it, we decompose $W$ as the union of sets $S_{i}$ given in terms of intersections only (basic semialgebraic sets) and repeatedly use Mayer-Vietoris sequences to recover the homology of $W$ from the homology of these pieces. This requires to consider a family of algebraic neighborhoods for each $S_{i}$ and to use homological algebra to establish isomorphisms between the homology groups of the $S_{i}$, those of their algebraic neighborhoods, and those of their coverings $\mathcal{U}_{i}$. One key ingredient to make this possible is the Semialgebraic Triangulation Theorem. Another key ingredient is ensuring that all the algebraic neighborhoods above are homotopically equivalent to their corresponding $S_{i}$ and probably the major technical effort in our agenda is to estimate a size (or tolerance) for these algebraic neighborhoods that guarantees this equivalence. We do this (in Section 4) in terms of the condition number. Our estimate quantifies the results of Durfee [32]. Its proof relies on an explicit construction of Whitney stratifications and submersions for which Thom's First Isotopy Lemma [63] can be applied. This use of semialgebraic geometry, as well as of differential and algebraic topology, sets our arguments apart from those in [29] and [15].

Future work. We are currently working in two directions extending this paper. On the one hand, to design an algorithm that works for arbitrary (i.e., not necessarily closed and given by lax formulas) semialgebraic sets. On the other hand, to show that the exponential weak complexity holds for a class of probability distributions more general than the ones we consider here. 


\section{Contents}

1 Introduction 1

2 Overview of the Algorithm $\quad 5$

2.1 Boolean combinations and propositional logic . . . . . . . . . . . . . . 6

2.2 Homogenization . . . . . . . . . . . . . . . . . . . . 6

2.3 Estimation of the condition number . . . . . . . . . . . . . . . . 7

2.4 Homologically equivalent complexes . . . . . . . . . . . . . . . . . . . 7

2.5 Algebraic neighborhoods of spherical semialgebraic sets . . . . . . . . . . . . . . 9

2.6 Computation of homology groups . . . . . . . . . . . . . . . . . . . . . . . . . . . . . . . . . . . . .

2.7 Probabilistic estimates . . . . . . . . . . . . . . . . . . 10

3 Condition and stability of the description $\quad 10$

3.1 The Weyl inner product . . . . . . . . . . . . . . . . . . . 10

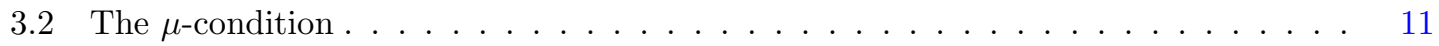

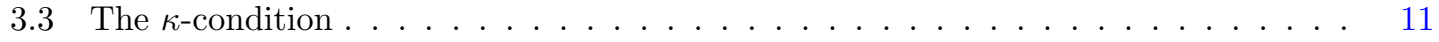

3.4 The intersection condition . . . . . . . . . . . . . . . . . . . 12

4 Geometry 14

4.1 Mather-Thom theory . . . . . . . . . . . . . . . . . . . . . 14

4.2 Semialgebraic Whitney stratifications of algebraic neighborhoods . . . . . . . 16

4.3 Trivializing charts and semilinear stratifications . . . . . . . . . . . . . . 18

5 Topology 22

5.1 Explicit Homological Nerve Theorem . . . . . . . . . . . . . . . . . . . . . . . 22

5.2 Homological Inclusion-Exclusion Transfer . . . . . . . . . . . . . . . . . . . . . . . 24

6 Algorithms 26

6.1 Grids . . . . . . . . . . . . . . . . . . . . . . . 26

6.2 Estimating the condition . . . . . . . . . . . . . . . . . . 26

6.3 Computation of simplicial complexes . . . . . . . . . . . . . . . . . . . 28

6.4 Computation of homology groups . . . . . . . . . . . . . . . . . 33

7 Affine Condition, Random Data and Proof of the Main Result 33

7.1 Affine intersection condition . . . . . . . . . . . . . . . . . . . . . . . . . . . . . . . . 33

7.2 Random tuples in $\mathcal{P}_{\boldsymbol{d}}[q]$ and $\mathcal{H}_{\boldsymbol{d}}[q] \ldots \ldots \ldots \ldots \ldots$

7.3 Proof of the Main Result . . . . . . . . . . . . . . . . . . . . . . . . . 35

7.4 Parallel computations . . . . . . . . . . . . . . . . . . 36

A On the smoothness assumption in Thom's first isotopy lemma 40

Acknowledgments. We are grateful to Saugata Basu, Pierre Lairez and Nicolai Vorobjov for helpful discussions.

\section{Overview of the Algorithm}

In this section we describe, with broad strokes, the various steps and ingredients of algorithm Homology. 


\subsection{Boolean combinations and propositional logic}

There is a close relationship between lax Boolean combinations of equalities and inequalities and propositional logic. Indeed, any such Boolean combination over $p \in \mathcal{P}_{\boldsymbol{d}}[q]$ corresponds to a propositional formula $\Phi$ over $3 q$ propositional variables $w_{j}^{\alpha_{j}}, \propto_{j} \in\{\leq,=, \geq\}$, $j \in\{1, \ldots, q\}$, built using the connectives $\vee$ and $\wedge$. Our Boolean combination is obtained by replacing $w_{j}^{\alpha_{j}}$ by $\left\{p_{j}(x) \propto_{j} 0\right\}$ as well as $\vee$ by $\cup$ and $\wedge$ by $\cap$. We will freely use this correspondence all along this paper. In particular, we will use the expression lax formula over $p \in \mathcal{P}_{\boldsymbol{d}}[q]$ to denote a propositional formula as described above.

A class of formulas of particular importance is that of purely conjunctive (usually referred to as clauses in the context of mathematical logic). Such formulas have the form $\bigwedge v_{i}$ where $v_{i}$ is either a variable or the negation of a variable (in the case of lax formulas only the first case is possible). Subsets of $\mathbb{R}^{n}$ defined by formulas of this kind over tuples $p \in \mathcal{P}_{\boldsymbol{d}}[q]$ are called basic semialgebraic.

A formula $\Psi$ is said to be in Disjunctive Normal Form (or in DNF for short) when it has the form

$$
\Psi \equiv \bigvee_{i \in I} \psi_{i}
$$

with $\psi_{i}$ purely conjunctive. It is a well-known fact (see, e.g., [42, Theorem 3 in Section 2.3]) that for every propositional formula $\Phi$ there exists an equivalent formula $\Psi$ in DNF in the same set of variables. The same holds true when restricted to lax formulas. In our context this implies that, for all $p \in \mathcal{P}_{\boldsymbol{d}}[q]$, the sets $W(p, \Phi)$ and $W(p, \Psi)$ coincide.

\subsection{Homogenization}

As before, let $\boldsymbol{d}=\left(d_{1}, \ldots, d_{q}\right)$ be a $q$-tuple of positive integers. We denote by $\mathcal{H}_{\boldsymbol{d}}[q]$ the vector space of $q$-tuples $f=\left(f_{1}, \ldots, f_{q}\right)$ of homogeneous polynomials $f_{i} \in \mathbb{R}\left[X_{0}, X_{1}, \ldots, X_{n}\right]$ of degree $d_{i}$. We put $\boldsymbol{d}^{*}:=(1, \boldsymbol{d})$. The homogenization map $\mathbf{H}: \mathcal{P}_{\boldsymbol{d}}[q] \rightarrow \mathcal{H}_{\boldsymbol{d}^{*}}[q+1]$ is defined by

$$
p \mapsto \mathrm{H}(p):=\left(\|p\| X_{0}, p_{1}^{\mathrm{h}}, \ldots, p_{q}^{\mathrm{h}}\right),
$$

where $p_{i}^{\mathrm{h}}:=p_{i}\left(X_{1} / X_{0}, \ldots, X_{n} / X_{0}\right) X_{0}^{d_{i}}$ denotes the homogenization of $p_{i}$ and $\|p\|$ stands for the Weyl norm of the tuple $p$ defined in $\S 3.1$.

Any formula $\Phi$ over $f \in \mathcal{H}_{\boldsymbol{d}}[q]$ defines a semialgebraic subset $\mathrm{S}(f, \Phi)$ of the sphere $\mathbb{S}^{n}$. It will be convenient to call these sets spherical semialgebraic. In order to simplify the notation, we will also write $\mathrm{S}(f=0)$ etc. with the obvious meaning.

The following result relates, for $p \in \mathcal{P}_{\boldsymbol{d}}[q]$ and a formula $\Phi$ over $p$, the topology of the semialgebraic subset $W(p, \Phi)$ of the Euclidean space $\mathbb{R}^{n}$ with that of the intersection of the spherical semialgebraic subset $\mathrm{S}(\mathrm{H}(p), \Phi)$ with the halfspace $X_{0} \geq 0$. We note that such a result would be straightforward if one intersected with $X_{0}>0$ instead.

Proposition 2.1. Let $p \in \mathcal{P}_{\boldsymbol{d}}[q]$ such that $\bar{\kappa}_{\text {aff }}(p)<\infty$, let $\Phi$ be a lax formula over $p$ and denote by $\Phi^{\mathrm{h}}$ the formula over $\mathrm{H}(p) \in \mathcal{H}_{\boldsymbol{d}^{*}}[q+1]$ given by

$$
\Phi^{\mathrm{h}}:=\Phi\left(p_{1}^{\mathrm{h}}, \ldots, p_{q}^{\mathrm{h}}\right) \wedge\left(\|p\| X_{0} \geq 0\right) .
$$

Then the spaces $W(p, \Phi)$ and $\left.\mathrm{S}\left(\mathrm{H}(p), \Phi^{\mathrm{h}}\right)\right)$ are homotopically equivalent.

We will prove Proposition 2.1 in $§ 7.1$. It allows us to assume, in all that follows, that we are dealing with spherical semialgebraic sets given by Boolean combinations of homogeneous polynomials. We will freely use in this new context the terminology introduced in $\S 2.1$. 


\subsection{Estimation of the condition number}

In $\S 3.4$ we will associate a condition number $\bar{\kappa}(f) \in[1, \infty]$ to a tuple $f \in \mathcal{H}_{\boldsymbol{d}}[q]$ whose inverse measures how near are the intersections between the hypersurfaces given by $f$ from being non-transversal (the condition number $\bar{\kappa}_{\text {aff }}(p)$ in Theorem 1.1 is actually $\bar{\kappa}(\mathrm{H}(p))$ ). This condition number provides information on the geometry of every possible spherical semialgebraic set built from $f$. Tuples $f$ for which $\bar{\kappa}(f)=\infty$ are said to be ill-posed. They are precisely those tuples for which there exists a formula $\Phi$ such that arbitrary small perturbations of $f$ may change the topology of $\mathrm{S}(f, \Phi)$. The set $\bar{\Sigma}_{\boldsymbol{d}}[q]$ of ill-posed tuples has positive codimension in $\mathcal{H}_{\boldsymbol{d}}[q]$ and $\bar{\kappa}(f)$ estimates how far is $f$ from $\bar{\Sigma}_{\boldsymbol{d}}[q]$.

The first substantial computational effort performed by Homology is to estimate the condition number $\bar{\kappa}(f)$ of a tuple $f \in \mathcal{H}_{\boldsymbol{d}}[q]$. The following result, which we will prove in $\S 6.2$, deals with this task.

Proposition 2.2. There is an algorithm $\bar{\kappa}$-Estimate that, given $f \in \mathcal{H}_{\boldsymbol{d}}[q]$, returns a number $\mathrm{K}$ such that

$$
0.99 \bar{\kappa}(f) \leq \mathrm{K} \leq \bar{\kappa}(f)
$$

if $\bar{\kappa}(f)<\infty$, or loops forever otherwise. The cost of this algorithm is bounded by $(q n D \bar{\kappa}(f))^{\mathcal{O}(n)}$.

\subsection{Homologically equivalent complexes}

The master plan to compute the homology of $\mathrm{S}(f, \Phi)$ passes through computing a simplicial complex $\mathfrak{C}$ homologically equivalent to $\mathrm{S}(f, \Phi)$. In the basic case, that is, when $\Phi$ is purely conjunctive, the construction of $\mathfrak{C}$ is based on Theorem 2.3 below. Recall that the Hausdorff distance between two nonempty compact sets $W, V \subseteq \mathbb{R}^{n+1}$ is given by

$$
d_{H}(W, V):=\max \left\{\max _{v \in V} d(W, v), \max _{w \in W} d(w, V)\right\}
$$

where $d$ denotes Euclidean distance in $\mathbb{R}^{n+1}$. If either $V$ or $W$ is empty then we take $d_{H}(V, W):=\infty$. We denote by $B(x, r)$ the Euclidean open ball with center $x$ and radius $r$. Moreover, for a closed subset $X \subseteq \mathbb{S}^{n}$, we define the open $r$-neighborhood of $X$ in $\mathbb{R}^{n+1}$,

$$
\mathcal{U}(X, r):=\bigcup_{x \in X} B(x, r)
$$

Similarly, we denote by $\mathcal{U}_{\mathbb{S}}(X, r)$ the open (spherical) $r$-neighborhood of $X$ in $\mathbb{S}^{n}$, which is defined with respect to angular distance. Clearly,

$$
\mathcal{U}_{\mathbb{S}}(X, r) \subseteq \mathcal{U}(X, r)
$$

The following result [15, Theorems 2.8 and 4.12] is a variant of a seminal result by Niyogi, Smale, and Weinberger [48].

Theorem 2.3 (Basic Homotopy Witness Theorem). Let $f \in \mathcal{H}_{\boldsymbol{d}}[q]$ and $\phi$ be purely conjunctive. Moreover, let $\mathcal{X} \subseteq \mathbb{S}^{n}$ be a closed subset and $\varepsilon>0$ be such that

$$
3 d_{H}(\mathcal{X}, \mathrm{S}(f, \phi))<\varepsilon<\frac{1}{14 D^{\frac{3}{2}} \bar{\kappa}(f)} .
$$

Then the inclusion $\mathrm{S}(f, \phi) \hookrightarrow \mathcal{U}(\mathcal{X}, \varepsilon)$ is a homotopy equivalence. 
For a finite $\mathcal{X}$ satisfying the hypothesis of Theorem 2.3 we can take $\mathfrak{C}$ to be the $\check{C}$ ech complex $\check{\mathrm{C}}_{\varepsilon}(\mathcal{X})$ associated to $(\mathcal{X}, \varepsilon)$ whose $k$-faces, we recall [33, p. 60], are the sets of $k+1$ points $\left\{x_{0}, \ldots, x_{k}\right\} \subseteq \mathcal{X}$ such that $\cap_{i \leq k} B\left(x_{i}, \varepsilon\right) \neq \varnothing$. The Nerve Theorem [40, Corollary 4G.3] then states that the simplicial complex $\mathfrak{C}$ is homotopically equivalent to $\mathcal{U}(\mathcal{X}, \varepsilon)$ and, by Theorem 2.3 , to $\mathrm{S}(f, \phi)$ as well. To compute the homology of $\mathrm{S}(f, \phi)$ it is therefore enough to construct a pair $(\mathcal{X}, \varepsilon)$ satisfying the inequalities in Theorem 2.3, then build the complex $\mathfrak{C}$, and finally compute the homology of $\mathfrak{C}$.

The retraction in the proof of Theorem 2.3 is a nearest-point retraction. As we saw in the Introduction, these retractions do not necessarily exist for arbitrary closed semialgebraic sets. This is why we won't attempt to obtain a complex homotopically equivalent to $\mathrm{S}(f, \Phi)$. Instead, we will show that an appropriate Boolean combination of Čech complexes achieves homological equivalence. We briefly describe how this is done.

Fix a finite set of points $\mathcal{G}$ in $\mathbb{S}^{n}$ and $\varepsilon>0$. For $\mathcal{X}_{1}, \mathcal{X}_{2} \subseteq \mathcal{G}$ we define the intersection $\check{\mathrm{C}}_{\varepsilon}\left(\mathcal{X}_{1}\right) \cap \check{\mathrm{C}}_{\varepsilon}\left(\mathcal{X}_{2}\right)$ to be the simplicial complex whose $k$-faces are the sets of points $\left\{x_{0}, \ldots, x_{k}\right\}$ which are $k$-faces of both $\check{\mathrm{C}}_{\varepsilon}\left(\mathcal{X}_{1}\right)$ and $\check{\mathrm{C}}_{\varepsilon}\left(\mathcal{X}_{2}\right)$. It is clear that $\check{\mathrm{C}}_{\varepsilon}\left(\mathcal{X}_{1}\right) \cap$ $\check{\mathrm{C}}_{\varepsilon}\left(\mathcal{X}_{2}\right)=\breve{\mathrm{C}}_{\varepsilon}\left(\mathcal{X}_{1} \cap \mathcal{X}_{2}\right)$. Similarly, we define the union $\check{\mathrm{C}}_{\varepsilon}\left(\mathcal{X}_{1}\right) \cup \check{\mathrm{C}}_{\varepsilon}\left(\mathcal{X}_{2}\right)$ to be the simplicial complex whose $k$-faces are the sets of points $\left\{x_{0}, \ldots, x_{k}\right\}$ which are $k$-faces of either $\check{\mathrm{C}}_{\varepsilon}\left(\mathcal{X}_{1}\right)$ or $\check{\mathrm{C}}_{\varepsilon}\left(\mathcal{X}_{2}\right)$. We observe that, in contrast with the behavior for intersections, we now only have $\check{\mathrm{C}}_{\varepsilon}\left(\mathcal{X}_{1}\right) \cup \check{\mathrm{C}}_{\varepsilon}\left(\mathcal{X}_{2}\right) \subseteq \check{\mathrm{C}}_{\varepsilon}\left(\mathcal{X}_{1} \cup \mathcal{X}_{2}\right)$. The union complex is not necessarily a Cech complex over a subset of $\mathcal{G}$.

Given a lax formula $\Phi$ over $f \in \mathcal{H}_{\boldsymbol{d}}[q]$ and finite sets $\mathcal{X}_{j}^{\leq}, \mathcal{X}_{j}^{=}, \mathcal{X}_{j}^{\geq} \subseteq \mathbb{S}^{n}$, for $j=1, \ldots, q$, associated to the $3 q$ atomic sets

$$
\begin{aligned}
& S_{j}^{\leq}:=\mathrm{S}\left(f_{j} \leq 0\right), \\
& S_{j}^{=}:=\mathrm{S}\left(f_{j}=0\right), \\
& S_{j}^{\geq}:=\mathrm{S}\left(f_{j} \geq 0\right),
\end{aligned}
$$

we can then consider the simplicial complex $\Phi\left(\check{\mathrm{C}}_{\varepsilon}\left(\mathcal{X}_{1}^{\leq}\right), \ldots, \check{\mathrm{C}}_{\varepsilon}\left(\mathcal{X}_{q}^{\geq}\right)\right)$, recursively built from the $\check{\mathrm{C}}_{\varepsilon}\left(\mathcal{X}_{j}^{\propto_{j}}\right)$ in the same way $\mathrm{S}(f, \Phi)$ is built from the $S_{j}^{\propto_{j}}$.

The following result is our extension of Theorem 2.3. We will will prove it in $\S 6.3$.

Theorem 2.4 (Homology Witness Theorem). Let $f \in \mathcal{H}_{\boldsymbol{d}}[q]$ and $\varepsilon>0$. Moreover, for $j=1, \ldots, q$, let $\mathcal{X}_{j}^{\leq}, \mathcal{X}_{j}^{=}, \mathcal{X}_{j}^{\geq} \subseteq \mathbb{S}^{n}$ be closed subsets such that for all $j, \mathcal{X}_{j}^{\leq} \cap \mathcal{X}_{j}^{\geq}=\mathcal{X}_{j}^{=}$ and such that for all $J \subseteq\{1, \ldots, q\}$ and all $\propto \in\{\leq,=, \geq\}^{J}$, we have

$$
3 d_{H}\left(\cap_{j \in J} \mathcal{X}_{j}^{\propto_{j}}, \cap_{j \in J} S_{j}^{\propto_{j}}\right) \leq \varepsilon \leq \frac{1}{14 D^{\frac{3}{2}} \bar{\kappa}(f)} .
$$

Then, for all lax formulas $\Phi$ over $f$, the set $\mathrm{S}(f, \Phi)$ and the simplicial complex

$$
\mathfrak{C}=\Phi\left(\check{C}_{\varepsilon}\left(\mathcal{X}_{1}^{\leq}\right), \check{C}_{\varepsilon}\left(\mathcal{X}_{1}^{=}\right), \check{C}_{\varepsilon}\left(\mathcal{X}_{1}^{\geq}\right), \ldots, \check{C}_{\varepsilon}\left(\mathcal{X}_{q}^{\leq}\right), \check{C}_{\varepsilon}\left(\mathcal{X}_{q}^{=}\right), \check{C}_{\varepsilon}\left(\mathcal{X}_{q}^{\geq}\right)\right)
$$

have the same homology.

Remark 2.5. The techniques used to prove Theorem 2.3 rely on the notion of reach (or feature size) $\tau(X)$ of a closed set $X$ in Euclidean space [34, §4]. This so because a positive reach of $X$ guarantees a nearest-point retraction onto $X$ from sufficiently small neigborhoods of $X$ [48, Prop. 7.1], and a finite $\bar{\kappa}(f)$ guarantees a positive reach of $\mathbf{S}(f, \phi)$ for 
all purely conjunctive $\phi$ [15, Thm. 4.12]. Unfortunately, for sets $X$ as the one drawn in the Introduction, the reach is zero. One may think that the use of the weak feature size $\tau_{w}$ and its associated retractions (see [20,22]) could be an appropriate replacement of the reach for arbitrary closed semialgebraic sets, since $\tau_{w}$ is guaranteed to be positive on these sets. However, the proof of this positivity (see [21]) does not give any effective way of bounding $\tau_{w}$. Indeed, it is still an open problem to bound $\tau_{w}\left(\cup_{i=1}^{m} S_{i}\right)$ in terms of the $\tau_{w}\left(S_{i}\right)$ and of geometric quantities capturing the relative position of the $S_{i}$.

\subsection{Algebraic neighborhoods of spherical semialgebraic sets}

Theorem 2.4 ensures that a collection of point clouds (finite sets of points) $\left\{\mathcal{X}_{i}^{\propto_{i}}\right\}$ sufficiently near to the sets $S_{i}^{\alpha_{i}}$ allows us to build a simplicial complex homologically equivalent to $\mathrm{S}(f, \Phi)$. The difficulty we now face is, given a candidate set $\mathcal{X}_{i}^{\alpha_{i}}$, how to estimate the Hausdorff distance between $\mathcal{X}_{i}^{\propto_{i}}$ and $S_{i}^{\propto_{i}}$. It was to solve this problem that algebraic neighborhoods were introduced in [15].

Algebraic neighborhoods of closed semialgebraic sets are obtained by relaxing the equalities and inequalities in their description. More concretely, given a lax formula $\Phi$ over $f \in \mathcal{H}_{\boldsymbol{d}}[q]$, the algebraic neighborhood $\mathrm{S}_{r}(f, \Phi)$ of $\mathrm{S}(f, \Phi)$ with tolerance $r$ is the spherical set defined by replacing the atoms $f_{i}=0$ by $\left|f_{i}(x)\right| \leq r\left\|f_{i}\right\|$, the atoms $f_{i} \geq 0$ by $f_{i}(x) \geq-r\left\|f_{i}\right\|$ and the atoms $f_{i} \leq 0$ by $f_{i}(x) \leq r\left\|f_{i}\right\|$. The open algebraic neighborhood $\mathrm{S}_{r}^{\circ}(f, \Phi)$ of $\mathrm{S}(f, \Phi)$ with tolerance $r$ is similarly defined but with strict inequalities.

A crucial difference between $r$-neighborhoods and algebraic neighborhoods of $\mathrm{S}(f, \Phi)$ is that, for a given $x \in \mathbb{S}^{n}$, it is computationally trivial to check membership to the latter and computationally expensive to do so for the former. But to use algebraic neighborhoods to bound Euclidean distances we need to understand how do $r$-neighborhoods and algebraic neighborhoods of $\mathbf{S}(f, \Phi)$ relate. The following inclusion is a simple consequence of the Exclusion Lemma (Lemma 3.1 in [26], see also [15, Prop. 4.17]) which goes back to [30]

$$
\mathcal{U}_{\mathbb{S}}(\mathrm{S}(f, \Phi), r) \subseteq \mathrm{S}_{D^{1 / 2} r}^{\circ}(f, \Phi),
$$

where, we recall, $D=\max _{i} \operatorname{deg} f_{i}$. An inclusion in the other direction, now involving the condition of $f$, was shown in [15, Thm. 4.19].

Proposition 2.6. Let $f \in \mathcal{H}_{\boldsymbol{d}}[q]$ and $r>0$ be such that $13 D^{3 / 2} \bar{\kappa}(f)^{2} r<1$. Then, for every purely conjunctive formula $\phi$ over $f$,

$$
\mathrm{S}_{r}^{\circ}(f, \phi) \subseteq \mathcal{U}_{\mathbb{S}}(\mathrm{S}(f, \phi), 3 \bar{\kappa}(f) r) .
$$

Proposition 2.6, together with the choice of the $\mathcal{X}_{i}^{\alpha_{i}}$ from a grid $\mathcal{G}$ sufficiently dense in $\mathbb{S}^{n}$, allows one to certify, in an efficient manner, that the hypothesis of Theorem 2.4 is satisfied. To prove this theorem, in addition, the following fundamental property of algebraic neighborhoods is used.

Theorem 2.7 (Quantitative Durfee's Theorem). Let $f \in \mathcal{H}_{\boldsymbol{d}}[q]$ and $r>0$ be such that $\sqrt{2} \bar{\kappa}(f) r<1$. Then, for every purely conjunctive lax formula $\phi$ over $f$, the inclusions in

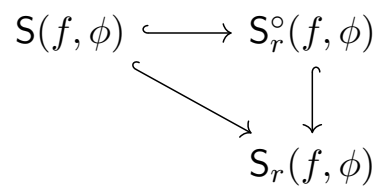

are homotopy equivalences.

We will devote all of Section 4 to prove Theorem 2.7. 


\subsection{Computation of homology groups}

Once in the possession of the complex $\mathfrak{C}$ homologically equivalent to $\mathrm{S}(f, \Phi)$, the computation of the homology of the latter reduces to doing so for the former. Algorithmic procedures for this task are well-known. We briefly describe them (and recall their complexity) in $\S 6.4$.

\subsection{Probabilistic estimates}

The primary complexity analysis of the algorithm Homology is condition-based. Cost bounds on input $(p, \Phi)$ depend on the condition $\bar{\kappa}_{\text {aff }}(p)$ (or on $\bar{\kappa}(f)$, as in the statement of Proposition 2.2). This complexity analysis takes form in part (i) of Theorem 1.1.

To obtain parts (ii) and (iii) one needs to estimate the probability tail of $\bar{\kappa}_{\text {aff }}(p)$. To do so, we will bound $\bar{\kappa}_{\text {aff }}(p)$ in terms of the normalized distance from $p$ to the set $\bar{\Sigma}_{\boldsymbol{d}}^{\mathrm{aff}}[q]:=\mathrm{H}^{-1}\left(\bar{\Sigma}_{\boldsymbol{d}}[q]\right) \subseteq \mathcal{P}_{\boldsymbol{d}}[q]$ of ill-posed tuples. This set is included in an algebraic cone $V$ of codimension 1 whose degree is bounded by an explicit function of $n, D$ and $q$. The tail $\operatorname{Prob}_{p \in \mathcal{P}_{\boldsymbol{d}}[q]}\left\{\bar{\kappa}_{\text {aff }}(p) \geq t\right\}$ is consequently bounded in terms of the volume of the $\frac{1}{t}$-neighborhood of $V \cap \mathbb{S}\left(\mathcal{P}_{\boldsymbol{d}}[q]\right)$. A general result estimating this volume in terms of $N:=\operatorname{dim} \mathcal{P}_{\boldsymbol{d}}[q]$, the degree of $V$, and its codimension is given in [16]. We employ this result to estimate the tail of $\bar{\kappa}_{\text {aff }}$ and use this estimate to obtain the desired weak cost bounds. This is carried out in Section 7 .

\section{Condition and stability of the description}

Our algorithm's design and analysis are condition-based. To carry them out we will define an appropriate condition number, $\bar{\kappa}(f)$, and show some of its main properties. This quantity follows a lineage of condition numbers (for different problems) going back to von Neumann and Goldstine [47] and Turing [64].

We begin endowing the vector space $\mathcal{H}_{\boldsymbol{d}}[q]$ with an inner product.

\subsection{The Weyl inner product}

For two homogeneous polynomials $g=\sum_{\alpha} g_{\alpha} X^{\alpha}$ and $h=\sum_{\alpha} h_{\alpha} X^{\alpha}$ of degree $d$, the Weyl inner product is given by

$$
\langle g, h\rangle:=\sum_{\alpha}\left(\begin{array}{l}
d \\
\alpha
\end{array}\right)^{-1} g_{\alpha} h_{\alpha}
$$

where $\left(\begin{array}{l}d \\ \alpha\end{array}\right):=\frac{d !}{\alpha_{0} ! \cdots \alpha_{d} !}$ is the multinomial coefficient. We extend this to pairs $f, f^{\prime} \in \mathcal{H}_{\boldsymbol{d}}[q]$ in the usual way,

$$
\left\langle f, f^{\prime}\right\rangle:=\sum_{i=1}^{q}\left\langle f_{i}, f_{i}^{\prime}\right\rangle .
$$

The most important feature of this inner product is that it is invariant under orthogonal changes of coordinates, i.e., that for each $u \in \mathscr{O}(n+1)$ and $f, g \in \mathcal{H}_{d}[q],\langle f, g\rangle=\langle f \circ$ $u, g \circ u\rangle$. In addition, it is the only inner product in $\mathcal{H}_{\boldsymbol{d}}[q]$ invariant under orthogonal transformations, up to renormalization in each component of $\mathcal{H}_{\boldsymbol{d}}[q]$, that extends to a Hermitian inner product invariant under unitary transformations in the complex analog of $\mathcal{H}_{\boldsymbol{d}}[q] ;$ cf. [14, Rem. 16.4]. 
The definition of the Weyl inner product in $\mathcal{H}_{\boldsymbol{d}}[q]$ naturally translates to $\mathcal{P}_{\boldsymbol{d}}[q]$ : for $p, s \in \mathcal{P}_{\boldsymbol{d}}[q]$ we define $\langle p, s\rangle:=\left\langle p^{\mathrm{h}}, s^{\mathrm{h}}\right\rangle$, where homogeneization is componentwise.

\subsection{The $\mu$-condition}

We will look at elements $f \in \mathcal{H}_{\boldsymbol{d}}[q]$ as polynomial maps $f: \mathbb{S}^{n} \rightarrow \mathbb{R}^{q}$. For $x \in \mathbb{S}^{n}$, we will denote by $\mathrm{D}_{x} f$ the tangent map $\mathrm{T}_{x} \mathbb{S}^{n} \rightarrow \mathbb{R}^{q}$. This is nothing more than the restriction to the linear subspace $\mathrm{T}_{x} \mathbb{S}^{n}$ of the usual derivative map of $f$ at $x$.

The $\mu$-condition of $f$ at $x \in \mathbb{S}^{n}$ is given by

$$
\mu(f, x):=\|f\|\left\|\mathrm{D}_{x} f^{\dagger} \Delta\right\|,
$$

where $\mathrm{D}_{x} f^{\dagger}$ is the Moore-Penrose inverse of $\mathrm{D}_{x} f, \Delta$ is the normalization matrix given by

$$
\Delta:=\left(\begin{array}{ccc}
\sqrt{d_{1}} & & \\
& \ddots & \\
& & \sqrt{d_{q}}
\end{array}\right),
$$

and the norm $\left\|\mathrm{D}_{x} f^{\dagger} \Delta\right\|$ is the spectral norm. By convention, we take $\mu(f, x)$ to be $\infty$ when $\mathrm{D}_{x} f$ is not surjective. One should see the inverse of $\mu(f, x)$ as a measure of how near from being non-surjective the tangent map $\mathrm{D}_{x} f$ is. The extra parameters $\|f\|$ and $\Delta$ are there to ensure scalability as well as the equalities in Theorem 3.4.

Remark 3.1. The $\mu$ condition number was introduced by Shub and Smale in their "Bézout series" $[54,55,56,58,57]$. It plays a crucial role in the solution of Smale's 17th problem [60, $6,13,45]$. The version $\mu(f, x)$ slightly differs from the one in these references; it is instead the minor variation introduced as $\mu_{\text {proj }}$ in [15] which allows, as shown in [15], an elegant Condition Number Theorem (Theorem 3.4 below).

\subsection{The $\kappa$-condition}

The quantity $\mu(f, x)$ is a good measure of how well-conditioned a zero $x$ of $f$ is. A large value of $\mu(f, x)$ when $f(x) \neq 0$ indicates, when working over the complex numbers, that there exists a small perturbation of $f$ having an ill-posed zero. The fact that this is no longer true in the real case led to the introduction, in [25], of the following condition number.

Definition 3.2. We define the real homogeneous condition number of $f \in \mathcal{H}_{\boldsymbol{d}}[q]$ at $x \in \mathbb{S}^{n}$ as

$$
\kappa(f, x):=\frac{1}{\sqrt{\frac{1}{\mu(f, x)^{2}}+\frac{\|f(x)\|^{2}}{\|f\|^{2}}}},
$$

where we use the usual conventions of infinity together with $\infty^{-1}=0$ and its reciprocal. We further define the real homogeneous condition number of $f$ by

$$
\kappa(f):=\max _{x \in \mathbb{S}^{n}} \kappa(f, x) .
$$

Remark 3.3. For $q>n$ and $f \in \mathcal{H}_{\boldsymbol{d}}[q]$, the system $f=0$ is overdetermined. This implies that $\mathrm{D}_{x} f$ cannot be surjective at any $x \in \mathbb{S}^{n}$ : for all $x \in \mathbb{S}^{n}, \mu(f, x)=\infty$ and $\kappa(f, x)=$ $\frac{\|f\|}{\|f(x)\|}$. In particular, $\kappa(f)<\infty$ if and only if $\mathrm{S}(f=0)$ is empty. 
The condition number $\kappa$ satisfies a Condition Number Theorem. That is, its inverse tells us how near $f \in \mathcal{H}_{\boldsymbol{d}}[q]$ is from being ill-posed. To be precise, note that $\kappa(f)=\infty$ if and only if there is some $x \in \mathrm{S}(f=0)$ such that $\mathrm{D}_{x} f$ is not surjective. This motivates to define the set of systems ill-posed at $x \in \mathbb{S}^{n}$ as

$$
\Sigma_{\boldsymbol{d}}[q]_{x}:=\left\{f \in \mathcal{H}_{\boldsymbol{d}}[q] \mid f(x)=0 \text { and } \mathrm{D}_{x} f \text { is not surjective }\right\},
$$

and the set of ill-posed systems as $\Sigma_{\boldsymbol{d}}[q]:=\bigcup_{x \in \mathbb{S}^{n}} \Sigma_{\boldsymbol{d}}[q]_{x}$. One should notice that $f \notin \Sigma_{\boldsymbol{d}}[q]$ if and only if 0 is a regular value of $f$ which, by the Implicit Function Theorem, is enough to guarantee that $\mathrm{S}(f=0)$ is smooth. The Condition Number Theorem for $\kappa$ is then the following.

Theorem 3.4. [15, Theorem 2.19] For all $f \in \mathcal{H}_{\boldsymbol{d}}[q]$ and $x \in \mathbb{S}^{n}$,

$$
\kappa(f, x)=\frac{\|f\|}{d\left(f, \Sigma_{\boldsymbol{d}}[q]_{x}\right)} \quad \text { and } \quad \kappa(f)=\frac{\|f\|}{d\left(f, \Sigma_{\boldsymbol{d}}[q]\right)},
$$

where $d$ is the distance induced by the Weyl inner product.

Corollary 3.5. For every $f \in \mathcal{H}_{\boldsymbol{d}}[q]$ and $x \in \mathbb{S}^{n}, \kappa(f, x) \geq 1$.

The following bound on $\mu$ in terms of $\kappa$ relates the values of $\mu$ and $\kappa$ near the zero set. It provides an important guarantee of the surjectivity of $\mathrm{D}_{x} f$.

Proposition 3.6. If $f \in \mathcal{H}_{\boldsymbol{d}}[q], x \in \mathbb{S}^{n}$ and $\sqrt{2} \kappa(f, x) \frac{\|f(x)\|}{\|f\|}<1$, then

$$
\mu(f, x) \leq \sqrt{2} \kappa(f, x) .
$$

Moreover, $\mathrm{D}_{x} f$ is surjective.

Proof. By the definition of $\kappa$,

$$
\frac{1}{\kappa(f, x)^{2}}=\frac{1}{\mu(f, x)^{2}}+\frac{\|f(x)\|^{2}}{\|f\|^{2}} \leq 2 \max \left\{\frac{1}{\mu(f, x)^{2}}, \frac{\|f(x)\|^{2}}{\|f\|^{2}}\right\} .
$$

Since $2 \frac{\|f(x)\|^{2}}{\|f\|^{2}}<\frac{1}{\kappa(f, x)^{2}}$ by hypothesis, we have $\max \left\{\frac{1}{\mu(f, x)^{2}}, \frac{\|f(x)\|^{2}}{\|f\|^{2}}\right\}=\frac{1}{\mu(f, x)^{2}}$ and the desired inequality follows. Finally, we note that $\mu(f, x)$ is finite if and only if $\mathrm{D}_{x} f^{\dagger}$ is defined if and only if $\mathrm{D}_{x} f$ is surjective.

\subsection{The intersection condition}

Assume a perturbation of the coefficients of $f$ changes the topology of $\mathrm{S}(f, \Phi)$. Then, along the way in this perturbation, a singularity must occur in some boundary of $\mathbf{S}(f, \Phi)$. Because of this, it is in the description of the boundary pieces where the condition for computing this topology lies.

The Zariski closure of one such boundary piece is given by some polynomial equalities. We note though that, once we have $n+1$ such equalities, the intersection will have to be empty to be well-posed, and so, there is no need to consider intersections of more than $n+1$ polynomials. This suggests the following definition. 
Definition 3.7. Given $f \in \mathcal{H}_{\boldsymbol{d}}[q]$, the real homogeneous intersection condition number of $f$ is defined as

$$
\bar{\kappa}(f):=\max \left\{\kappa\left(f^{L}\right)|L \subseteq\{1, \ldots, q\},| L \mid \leq n+1\right\}
$$

where $f^{L}:=\left(f_{i}\right)_{i \in L}$.

The following result explains the name "intersection condition" as it shows that, in some sense, the inverse of $\bar{\kappa}$ measures how near are the intersections between the hypersurfaces given by $f$ from being non-transversal.

Theorem 3.8. Let $f \in \mathcal{H}_{\boldsymbol{d}}[q]$. Then $\bar{\kappa}(f)$ is finite if and only if 0 is a regular value of each $f_{i}$, i.e., for every $x \in \mathrm{S}\left(f_{i}=0\right)$, the map $\mathrm{D}_{x} f_{i}: \mathrm{T}_{x} \mathbb{S}^{n} \rightarrow \mathbb{R}$ is surjective, and any intersection between the smooth subvarieties $\mathrm{S}\left(f_{i}=0\right)$ is transversal, i.e., for all $I \subseteq\{1, \ldots, q\}$ and all $x \in \bigcap_{i \in I} \mathrm{~S}\left(f_{i}=0\right)$,

$$
\sum_{i \in I} \operatorname{codim}_{\mathrm{T}_{x} \mathbb{S}^{n}} \mathrm{~T}_{x}\left(\mathrm{~S}\left(f_{i}=0\right)\right)=\operatorname{codim}_{\mathrm{T}_{x} \mathbb{S}^{n}} \bigcap_{i \in I} \mathrm{~T}_{x}\left(\mathrm{~S}\left(f_{i}=0\right)\right) .
$$

Sketch of the proof. It is clear that $\bar{\kappa}(f)$ is finite if and only if for every $L \subseteq\{1, \ldots, q\}$ of size at most $n+1$, the map $\mathrm{D}_{x} f^{L}: \mathrm{T}_{x} \mathbb{S}^{n} \rightarrow \mathbb{R}^{L}$ is surjective for each $x \in \mathrm{S}\left(\bigwedge_{i \in L}\left(f_{i}=0\right)\right)$. Now, this will happen if and only if the hyperplanes ker $\mathrm{D}_{x} f_{i}$ of $\mathrm{T}_{x} \mathbb{S}^{n}$ intersect transversally, but this is exactly the claimed equality as ker $\mathrm{D}_{x} f_{i}=\mathrm{T}_{x} \mathrm{~S}\left(f_{i}=0\right)$.

Remark 3.9. Consider a purely conjunctive formula

$$
\bigwedge_{I \in I}\left(f_{i}=0\right) \wedge \bigwedge_{j \in J}\left(f_{j} \propto_{j} 0\right)
$$

where $\propto_{j} \in\{\leq, \geq\}$ and $I, J \subseteq\{1, \ldots, q\}$ with $I \cap J=\varnothing$. A condition number $\kappa_{*}\left(f_{I}, f_{J}\right)$, now depending on both $f$ and $\phi$ was defined in [15] as follows,

$$
\kappa_{*}\left(f_{I}, f_{J}\right):=\max _{\substack{L \subseteq J \\|L| \leq n-|I|+1}} \kappa\left(f_{I \cup L}\right)
$$

where $f_{I}=\left(f_{i}\right)_{i \in I}$ and similarly for $f_{J}$ and $f_{I \cup L}$. It is immediate to verify that $\bar{\kappa}(f)=$ $\kappa_{*}(\varnothing, f)$ and that

$$
\kappa_{*}\left(f_{I}, f_{J}\right) \leq \bar{\kappa}(f) .
$$

These relations allow us to use the bounds in [15] replacing $\kappa_{*}$ by $\bar{\kappa}$ in them.

As a first application of the remark above we note that, although we don't have an exact Condition Number Theorem for $\bar{\kappa}$, we do have a bound. The following result is an immediate consequence of [15, Theorem 4.10].

Theorem 3.10. For all $f \in \mathcal{H}_{\boldsymbol{d}}[q]$ we have

$$
\bar{\kappa}(f) \leq \frac{\|f\|}{d\left(f, \bar{\Sigma}_{\boldsymbol{d}}[q]\right)},
$$

where

$$
\bar{\Sigma}_{\boldsymbol{d}}[q]:=\bigcup\left\{\Sigma_{\boldsymbol{d}}[q]_{L}|L \subseteq\{1, \ldots, q\},| L \mid \leq n+1\right\}
$$

with

$$
\Sigma_{\boldsymbol{d}}[q]_{L}:=\left\{f \in \mathcal{H}_{\boldsymbol{d}}[q]\left|\exists \xi \in \bigcap_{i \in L} \mathrm{~S}\left(f_{i}=0\right): \operatorname{rank} \mathrm{D}_{\xi} f^{L}<\right| L \mid\right\}
$$

and $d$ is the distance induced by the inner product of $\mathcal{H}_{\boldsymbol{d}}[q]$. 


\section{Geometry}

The main goal of this section is to prove Quantitative Durfee's Theorem 2.7.

\subsection{Mather-Thom theory}

Let us start with a motivation. Gradient retractions are central in Morse theory, where they are used to establish homotopy equivalences between fibers of Morse functions at pairs of regular values without critical values in between. More precisely, it is known that for a submersion $\alpha: \mathcal{M} \rightarrow I$ from a compact manifold $\mathcal{M}$ to an interval $I \subseteq \mathbb{R}$, the gradient of $\alpha$ induces a homotopy equivalence $\alpha^{-1}(t) \subseteq \alpha^{-1}(J)$ for any subinterval $J \subseteq I$ and any $t \in J$. In more general terms, but also using the gradient of $\alpha$ to prove it, this translates into the following statement (a particular case of Ehresmann's Lemma): for a submersion $\alpha: \mathcal{M} \rightarrow I$ from a compact manifold $\mathcal{M}$ to an interval $I \subseteq \mathbb{R}$, the map $\alpha: \mathcal{M} \rightarrow I$ is a trivial fiber bundle. Recall that a trivial fiber bundle $\alpha: E \rightarrow B$ is a continuous map of topological spaces for which there is a subspace $F$ of $E$ (the fiber) and a homeomorphism $h: E \rightarrow F \times B$ such that the diagram

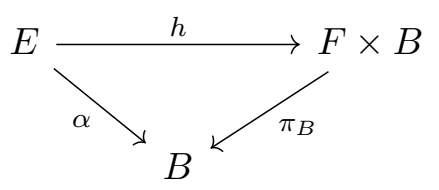

commutes. That is, $\alpha$ is a projection in disguise.

The extension of these results to a more general class of maps is part of the so-called stratified Mather-Thom theory [46], which allows one to generalize the results above from smooth to semialgebraic, not necessarily smooth, maps. We next outline the main notions of this theory (see also [35]).

The following definition generalizes the notion of a triangulation of $\mathcal{M}$, by allowing to decompose $\mathcal{M}$ into more general pieces.

Definition 4.1. [46, 35] A Whitney stratification of a smooth manifold $\mathcal{M}$ of dimension $m$ is a partition $\mathcal{S}$ of $\mathcal{M}$ into locally closed smooth submanifolds of $\mathcal{M}$, called strata, such that:

F (Locally finite) Every $x \in \mathcal{M}$ has a neighborhood intersecting finitely many strata only.

$\mathrm{W}$ (Whitney's condition b) For every strata $\varsigma, \sigma \in \mathcal{S}$, every point $x \in \varsigma \cap \bar{\sigma}$, every sequence of points $\left\{x_{\ell}\right\}_{\ell \in \mathbb{N}}$ in $\varsigma$ converging to $x$, and every sequence of points $\left\{y_{\ell}\right\}_{\ell \in \mathbb{N}}$ in $\sigma$ converging to $x$, we have that, in all local charts of $\mathcal{M}$ around $x$,

$$
\lim _{\ell \rightarrow \infty} \overline{x_{\ell}, y_{\ell}} \subseteq \lim _{\ell \rightarrow \infty} \mathrm{T}_{y_{\ell}} \sigma,
$$

provided both limits exist. The inclusion should be interpreted in the local coordinates of the chart: $\overline{x_{\ell}, y_{\ell}}$ denotes the straight line joining $x_{\ell}$ and $y_{\ell}, \mathrm{T}_{y_{\ell}} \sigma$ denotes the affine plane tangent to $\sigma$ at $y_{\ell}$, and the limits are to be interpreted in the corresponding Grassmannians of $\mathbb{R}^{m}$.

Remark 4.2. It is usual for the definition of Whitney stratification to include the so-called boundary condition which states that for every pair of strata $\varsigma, \sigma \in \mathcal{S}, \varsigma \cap \bar{\sigma} \neq \varnothing$ implies $\varsigma \subseteq \bar{\sigma}$. We omit it from the definition, because, as shown in [35, p. 16], this condition is not needed. 
A few comments are in order. Clearly, every smooth manifold $\mathcal{M}$ has the obvious Whitney stratification $\{\mathcal{M}\}$. Also, according to [46, Lemma 2.2], it is sufficient to check Whitney's condition b in a local chart. As for condition F, we won't mention it in what follows as we will only deal with finite stratifications.

The necessity of Whitney's condition $b$ is demonstrated by the following well-known example. Consider the stratification of $\mathbb{R}^{2}$ consisting of the point $\{0\}$, the smooth onedimensional submanifold

$$
C:=\left\{\left(e^{t} \cos (t), e^{t} \sin (t)\right) \mid t \in \mathbb{R}\right\},
$$

and the open subset $\sigma:=\mathbb{R}^{2} \backslash(\{0\} \cup C)$. Note that $C$ is a logarithmic spiral and that the angle between $\overline{0, x}$ and $\mathrm{T}_{x} C$ is $\pi / 4$ for all $x \in C$. This implies that

$$
\lim _{\ell \rightarrow \infty} \overline{0, y_{\ell}} \not \lim _{\ell \rightarrow \infty} \mathrm{T}_{y_{\ell}} C
$$

for all sequences $\left\{y_{\ell}\right\}$ of points in $C$, whenever the two limits of lines exist. Therefore, Whitney's condition $b$ is violated (take the constant sequence $\{0\}$ as the sequence $\left\{x_{\ell}\right\}$ ). Indeed, the purpose of condition b is to exclude wild variations such as the one of the logarithmic spiral when approaching the origin.

Whitney stratifications are closed under various operations.

Proposition 4.3. [35, Ch. I, (1.2) and (1.4)] Let $\mathcal{W}$ be a Whitney stratification of a smooth manifold $\mathcal{M}$.

(R) If $U$ is an open subset of $\mathcal{M}$, then $\mathcal{W}_{\mid U}:=\{\sigma \cap U \mid \sigma \cap U \neq \varnothing\}$ is a Whitney stratification of $U$.

(P) If $\mathcal{W}^{\prime}$ is a Whitney stratification of a smooth manifold $\mathcal{M}^{\prime}$, then $\mathcal{W} \times \mathcal{W}^{\prime}:=\left\{\sigma \times \sigma^{\prime} \mid\right.$ $\left.\sigma \in \mathcal{W}, \sigma^{\prime} \in \mathcal{W}^{\prime}\right\}$ is a Whitney stratification of $\mathcal{M} \times \mathcal{M}^{\prime}$.

Thom's first isotopy lemma [63] extends Ehresmann's Lemma to maps $\alpha: \mathcal{M} \rightarrow \mathbb{R}^{k}$ that are in a way compatible with a Whitney stratification of $\mathcal{M}$. Recall that a proper map is a continuous map for which the preimage of any compact set is compact.

Theorem 4.4 (Thom's first isotopy lemma). Let $\mathcal{M}$ be a smooth manifold with a Whitney stratification $\mathcal{S}$ and let $\alpha: \mathcal{M} \rightarrow \mathbb{R}^{k}$ be a continuous proper map such that:

- for each stratum $\sigma \in \mathcal{S}$, there is an open neighborhood $U$ of $\bar{\sigma}$ and a smooth map $\varphi: U \rightarrow \mathbb{R}^{k}$ such that $\alpha_{\mid \sigma}=\varphi$,

- for each stratum $\sigma \in \mathcal{S}, \alpha_{\mid \sigma}: \sigma \rightarrow \mathbb{R}^{k}$ is surjective,

- for each stratum $\sigma \in \mathcal{S}, \alpha_{\mid \sigma}: \sigma \rightarrow \mathbb{R}^{k}$ is a smooth submersion.

Then $\alpha$ is a trivial fiber bundle. In particular, for all subsets $U, V \subseteq \mathbb{R}^{k}, \alpha^{-1}(U) \subseteq \alpha^{-1}(V)$ is a homotopy equivalence whenever $U \subseteq V$ is so.

In the versions of Thom's first isotopy lemma we found in the literature, [46, Proposition 11.1] and [35, Ch. II, Theorem 5.2], the map $\alpha: \mathcal{M} \rightarrow \mathbb{R}^{k}$ is assumed to be smooth. We will show in Appendix A how Theorem 4.4 follows from the statement in [35]. 


\subsection{Semialgebraic Whitney stratifications of algebraic neighborhoods}

Our next result constructs a Whitney stratification for algebraic neighborhoods of basic semialgebraic sets that satisfies the hypothesis needed for applying Thom's first isotopy lemma.

We define the negative part of $t \in \mathbb{R}$ to be $|t|_{-}:=\max \{-t, 0\}$.

Proposition 4.5. Let $f \in \mathcal{H}_{\boldsymbol{d}}[q], \rho>0$ be such that $\sqrt{2} \bar{\kappa}(f) \rho<1$ and $\phi$ be the purely conjunctive lax formula

$$
\phi \equiv \bigwedge_{i \in E}\left(f_{i}=0\right) \wedge \bigwedge_{i \in P}\left(f_{i} \geq 0\right)
$$

with disjoint index sets $E, P$ such that $E \cup P=\{1, \ldots, q\}$. Consider the open subset $\mathcal{M}:=\mathrm{S}_{\rho}^{\circ}(f, \phi) \backslash \mathrm{S}(f, \phi)$ of $\mathbb{S}^{n}$ and the continuous map $\alpha: \mathcal{M} \rightarrow \mathbb{R}$ defined by

$$
\alpha(x):=\max \left\{\max _{i \in E} \frac{\left|f_{i}(x)\right|}{\left\|f_{i}\right\|}, \max _{j \in P} \frac{\left|f_{j}(x)\right|_{-}}{\left\|f_{j}\right\|}\right\} .
$$

Finally, for $K \subseteq E$ and $L \subseteq P$ define

$$
S_{K, L}:=\left\{\begin{array}{l|l}
x \in \mathcal{M} & \begin{array}{l}
\forall i \in E, i \in K \Leftrightarrow \alpha(x)=\left|f_{i}(x)\right| /\left\|f_{i}\right\| \\
\forall j \in P, j \in L \Leftrightarrow \alpha(x)=\left|f_{j}(x)\right|_{-} /\left\|f_{j}\right\|
\end{array}
\end{array}\right\} .
$$

Then the collection $\mathcal{W}:=\left\{S_{K, L} \mid S_{K, L} \neq \varnothing\right\}$ is a Whitney stratification of $\mathcal{M}$. Furthermore, for each stratum $S_{K, L}$,

(1) $S_{K, L}$ has codimension $|K|+|L|-1$,

(2) $\alpha_{\mid S_{K, L}}$ is a smooth submersion, and

(3) $\alpha\left(S_{K, L}\right)=(0, \rho)$.

We observe that, for $x \in \mathbb{S}^{n}$ and $r \geq 0, \alpha(x) \leq r$ if and only if $x \in \mathrm{S}_{r}(f, \phi)$. In particular, $\mathbf{S}(f, \phi)$ is the zero set of $\alpha$.

To avoid breaking the line of thought, we postpone the proof of Proposition 4.5 to $\S 4.3$ below and use it now to show the following, which is our last step before proving Theorem 2.7.

Proposition 4.6. Let $f \in \mathcal{H}_{\boldsymbol{d}}[q]$ and $r>0$ be such that $\sqrt{2} \bar{\kappa}(f) r<1$ and $\phi$ be a purely conjunctive lax formula. Then for all $r^{\prime} \in(0, r)$, the inclusions in

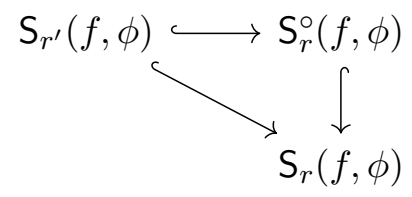

are homotopy equivalences.

Proof. After permuting the polynomials, changing signs and eliminating non-occurring polynomials, we can assume that

$$
\phi \equiv \bigwedge_{i \in E}\left(f_{i}=0\right) \wedge \bigwedge_{i \in P}\left(f_{i} \geq 0\right)
$$


where the union $E \cup P=\{1, \ldots, q\}$ is disjoint, since these operations do not increase the value of $\bar{\kappa}(f)$. Also, let $\rho>r$ be such that $\sqrt{2} \bar{\kappa}(f) \rho<1$.

Proposition 4.5 gives us a Whitney stratification of $\mathcal{M}=\mathrm{S}_{\rho}^{\circ}(f, \phi) \backslash \mathrm{S}(f, \phi)$ on which $\alpha: \mathcal{M} \rightarrow(0, \rho)$ is a proper map satisfying the hypothesis of Theorem 4.4. Notice that the first hypothesis is satisfied because on each $\overline{S_{K, L}}, \alpha$ agrees with the absolute value of a polynomial, which is smooth as long it does not vanish, which is guaranteed by the fact that $\alpha$ only takes positive values on $\Omega$. We can therefore use this theorem to deduce that $\alpha$ is a trivial fiber bundle. Let $F$ denote its fiber. Then there is a continuous map $\varphi: \mathcal{M} \rightarrow F$ such that

$$
h: \mathcal{M} \rightarrow F \times(0, \rho), x \mapsto(\varphi(x), \alpha(x))
$$

is a homeomorphism. Using this function, we see that the inclusion $\mathrm{S}_{r^{\prime}}(f, \phi) \hookrightarrow \mathrm{S}_{r}(f, \phi)$ is a homotopy equivalence by the following continuous retraction

$$
\begin{aligned}
\eta: \mathrm{S}_{r}(f, \phi) \times[0,1] & \rightarrow \mathrm{S}_{r}(f, \phi) \\
(x, t) & \mapsto \begin{cases}x & \text { if } x \in \mathrm{S}_{r^{\prime}}(f, \phi) \\
h^{-1}\left(\varphi(x), t r^{\prime}+(1-t) \alpha(x)\right) & \text { otherwise. }\end{cases}
\end{aligned}
$$

This restricts to a continuous retraction of $\mathrm{S}_{r}^{\circ}(f, \phi)$ onto $\mathrm{S}_{r^{\prime}}(f, \phi)$, which shows also that the inclusion $\mathrm{S}_{r^{\prime}}(f, \phi) \hookrightarrow \mathrm{S}_{r}^{\circ}(f, \phi)$ is a homotopy equivalence.

To show that the inclusion $\iota: \mathrm{S}_{r}^{\circ}(f, \phi) \hookrightarrow \mathrm{S}_{r}(f, \phi)$ is a homotopy equivalence, consider the retraction $\varrho: x \mapsto \eta(x, 1)$ of $\mathrm{S}_{r}(f, \phi)$ onto $\mathrm{S}_{r^{\prime}}(f, \phi)$ and its restriction $\varrho_{\circ}$ to a retraction of $\mathrm{S}_{r}^{\circ}(f, \phi)$ onto $\mathrm{S}_{r^{\prime}}(f, \phi)$. We have shown above, using the map $\eta$, that $\varrho$ and $\varrho_{\circ}$ are homotopic to the identity maps of $\mathrm{S}_{r}(f, \phi)$ and $\mathrm{S}_{r}^{\circ}(f, \phi)$, respectively. Hence $\iota$ is a homotopy equivalence, because both $\varrho \circ \iota=\varrho_{\circ}$ and $\iota \circ \varrho=\varrho$ are homotopic to the corresponding identity maps.

Remark 4.7. Notice that the proof of Proposition 4.6 cannot be extended to the case $r^{\prime}=0$, directly proving Theorem 2.7, because the hypotheses of Thom's first isotopy lemma don't apply if we include the zero set of $\alpha$ inside $\mathcal{M}$. But we can now proceed with the proof of this theorem.

Proof of Theorem 2.7. By Proposition 4.6 the inclusion $\mathrm{S}_{r}^{\circ}(f, \phi) \hookrightarrow \mathrm{S}_{r}(f, \phi)$ is a homotopy equivalence. It is therefore enough to show that so is $\mathrm{S}(f, \phi) \hookrightarrow \mathrm{S}_{r}(f, \phi)$, as the third equivalence is a consequence of these two.

By the Semialgebraic Triangulation Theorem [9, Theorem 9.2.1], $\mathrm{S}_{r}(f, \phi)$ has the structure of a CW complex of which $\mathrm{S}(f, \phi)$ is a subcomplex. Therefore, by [40, Prop. A.5.], there is an open neighborhood $U$ satisfying that $\mathrm{S}(f, \phi) \subseteq U \subseteq \mathrm{S}_{r}(f, \phi)$ and that $\mathrm{S}(f, \phi) \hookrightarrow U$ is a homotopy equivalence. Notice that $U$ is open in the sphere, because we can assume that $U \subseteq \mathrm{S}_{r}^{\circ}(f, \phi)$ by choosing it sufficiently small.

Because the family $\left\{\mathrm{S}_{\rho}(f, \varphi)\right\}_{\rho \in(0, r)}$ is a descending family of compact sets satisfying that $\cap_{\rho \in(0, r)} \mathrm{S}_{\rho}(f, \varphi)=\mathrm{S}(f, \phi)$, there exists a sufficiently small $r^{\prime} \in(0, r)$ such that $\mathrm{S}_{r^{\prime}}(f, \phi) \subseteq U$. This gives us the following sequence of inclusions

$$
\mathrm{S}(f, \phi) \hookrightarrow \mathrm{S}_{r^{\prime}}(f, \phi) \hookrightarrow U \hookrightarrow \mathrm{S}_{r}(f, \phi) .
$$

Passing to $k$ th homotopy groups we obtain the sequence of group homomorphisms

$$
\pi_{k}(\mathrm{~S}(f, \phi)) \stackrel{\alpha}{\longrightarrow} \pi_{k}\left(\mathrm{~S}_{r^{\prime}}(f, \phi)\right) \stackrel{\beta}{\longrightarrow} \pi_{k}(U) \stackrel{\gamma}{\longrightarrow} \pi_{k}\left(\mathrm{~S}_{r}(f, \phi)\right)
$$


where $\beta \circ \alpha$ is an isomorphism by the choice of $U$ and $\gamma \circ \beta$ is so due to Proposition 4.6. It follows that $\alpha, \beta$ and $\gamma$ are isomorphisms and, hence, so is $\gamma \circ \beta \circ \alpha$.

We have thus shown that the inclusion $\mathrm{S}(f, \phi) \hookrightarrow \mathrm{S}_{r}(f, \phi)$ induces an isomorphism of homotopy groups. This translates to a homotopy equivalence by virtue of the Semialgebraic Triangulation Theorem [9, Theorem 9.2.1] and Whitehead's Theorem [40, Theorem 4.5], which states that a continuous map of $\mathrm{CW}$ complexes that induces an isomorphism of homotopy groups is an homotopy equivalence.

Remark 4.8. In both Theorem 2.7 and Proposition 4.6 the inclusions (with the exception of $\left.\mathrm{S}_{r}(f, \phi) \hookrightarrow \mathrm{S}_{r}^{\circ}(f, \phi)\right)$ are actually deformation retractions. For Proposition 4.6, this can be seen by modifying our proof; for Theorem 2.7 , one can conclude using the stronger version of Whitehead's Theorem for subcomplexes [40, Theorem 4.5].

\subsection{Trivializing charts and semilinear stratifications}

The goal of this subsection is to prove Proposition 4.5. The overall idea of the proof relies on two stepping stones. Firstly, to show that, at each point $x$ of $\mathcal{M}$ we can define a local chart for which the normalized components $f_{i} /\left|f_{i}\right|$ of $f$ are the coordinate functions. Secondly, once with these local charts at hand, to show that the values taken by the normalized polynomials are enough to define the desired stratification. As these are values of coordinate functions, the resulting strata are semilinear.

These stepping stones are dealt with, respectively, by the two lemmas below. We begin with a simple consequence of the Implicit Function Theorem. Recall, $g^{S}:=\left(g_{i}\right)_{i \in S}$.

Lemma 4.9. For given $f \in \mathcal{H}_{\boldsymbol{d}}[q]$ put $g_{i}:=f_{i} /\left|f_{i}\right|$. Fix $x \in \mathbb{S}^{n}$, and let $r>0$ be such that $\sqrt{2} \bar{\kappa}(f) r<1$. We define the index set

$$
S:=\left\{i \in\{1, \ldots q\}|| g_{i}(x) \mid \leq r\right\}
$$

and set $\bar{u}:=g^{S}(x) \in \mathbb{R}^{S}$. Then $|S| \leq n$, and there exist an open neighborhood $O_{x}$ of $x$ in $\mathbb{S}^{n}$ and $\varepsilon>0$ with the following properties:

(t1) We have $\left|g_{i}(y)\right|>r$ for all $i \notin S$ and all $y \in O_{x}$.

(t2) For all $i$ such that $g_{i}(x) \neq 0$, the sign of $g_{i}$ does not change on $O_{x}$.

(t3) The set $\mathcal{Z}_{x}:=\left\{y \in O_{x} \mid f^{S}(y)=f^{S}(x)\right\}$ is a smooth submanifold of $\mathbb{S}^{n}$ of codimension $|S|$, and there exists a diffeomorphism $h$ such that the diagram

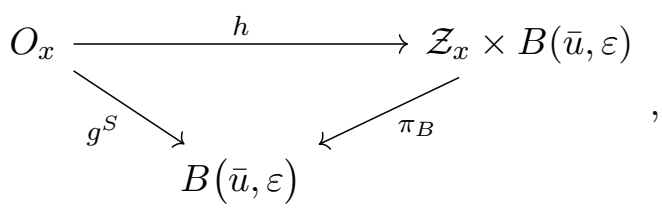

commutes (that is, for every $i \in S, g_{i}$ becomes a coordinate projection in the coordinates on $O_{x}$ given by $h$ ).

Proof. Assume first that $S$ is nonempty. Proposition 3.6 implies that $\mathrm{D}_{x} f^{S}$ is surjective, since $\sqrt{2} \kappa\left(f^{S}\right) \frac{\left\|f^{S}(x)\right\|}{\left\|f^{S}\right\|}<1$. So clearly $|S| \leq m$. Hence the derivative of the map $g^{S}$ at $x$ is surjective as well. The Implicit Function Theorem implies the existence of a diffeomorphism $h$ and a neighborhood $O_{x}$ satisfying (t3) with $\mathcal{Z}_{x}$ smooth. By shrinking $O_{x}$, we can guarantee the properties (t1) and (t2). Finally, the assertion is easily checked if $S$ is empty. 
We will call the pair $\left(O_{x}, h\right)$ a trivializing chart at $x$. We can describe a point $y \in O_{x}$ by its trivializing coordinates $(z, u) \in \mathcal{Z}_{x} \times B(\bar{u}, \varepsilon)$, where $u=\left(u_{i}\right)_{i \in S}$ and $h(y)=(z, u)$. In these coordinates, the normalized polynomial $g_{i}=f_{i} /\left\|f_{i}\right\|$, for $i \in S$, takes the form $(z, u) \mapsto u_{i}$.

Our second stepping stone establishes a Whitney stratification in a combinatorial situation, where all the strata are semilinear. The setting is as follows. Recall that $|t|_{-}=$ $\max \{-t, 0\}$ is the negative part of $t \in \mathbb{R}$.

Let $S=I \cup J$ be a partition of a nonempty finite set $S$. We associate with this partition the finite union of open halfspaces

$$
\Omega:=\bigcup_{i \in I}\left\{u \in \mathbb{R}^{S} \mid u_{i} \neq 0\right\} \cup \bigcup_{j \in J}\left\{u \in \mathbb{R}^{S} \mid u_{j}<0\right\} .
$$

Consider the function $\alpha: \mathbb{R}^{S} \rightarrow[0, \infty)$ defined by

$$
\alpha(u):=\max \left\{\max _{i \in I}\left|u_{i}\right|, \max _{j \in J}\left|u_{k}\right|_{-}\right\}
$$

and write $K_{u}:=\left\{i \in I|| u_{i} \mid=\alpha(u)\right\}, L_{u}:=\left\{\left.j \in J|| u_{j}\right|_{-}=\alpha(u)\right\}$ for the set of indices, where at $u \in \Omega, \alpha$ attains the maximum over $I$ and $J$, respectively. If we define

$$
\sigma_{K, L}:=\left\{u \in \Omega \mid K_{u}=K, L_{u}=L\right\}
$$

for a pair of subsets $K \subseteq I$ and $L \subseteq J$, we see that $\left\{\sigma_{K, L} \mid \sigma_{K, L} \neq \varnothing\right\}$ is a partition of $\Omega$. Also, it is easy to check that

$$
\sigma_{K, L}:=\left\{\begin{array}{l|l}
u \in \Omega \mid \begin{array}{l}
\forall i \in I, i \in K \Leftrightarrow \alpha(u)=\left|u_{i}\right| \\
\forall j \in J, j \in L \Leftrightarrow \alpha(u)=\left|u_{j}\right|_{-}
\end{array}
\end{array}\right\} .
$$

Lemma 4.10. In the above setting, $\mathcal{W}:=\left\{\sigma_{K, L} \mid \sigma_{K, L} \neq \varnothing\right\}$ is a Whitney stratification of $\Omega$. Furthermore, for each stratum $\sigma_{K, L}$ in $\mathcal{W}$ :

(1) $\sigma_{K, L}$ has codimension $|K|+|L|-1$,

(2) $\alpha_{\mid \sigma_{K, L}}$ is a smooth submersion, and

(3) if $\sigma_{K^{\prime}, L^{\prime}} \subseteq \overline{\sigma_{K, L}}$, then $\alpha\left(\sigma_{K^{\prime}, L^{\prime}}\right) \subseteq \alpha\left(\sigma_{K, L}\right)$.

We postpone the proof of this lemma (which is a long sequence of elementary arguments) and proceed with the proof of Proposition 4.5.

Proof of Proposition 4.5. By the locality of the definition of Whitney stratifications, in order to prove that a family of subsets $\mathcal{W}$ is a Whitney stratification of a manifold $\mathcal{M}$, it is enough to show that for every point $x \in \mathcal{M}$, there is an open neighborhood $O_{x}$ of $x$ in $\mathcal{M}$ such that $\mathcal{W} \cap O_{x}:=\left\{S \cap O_{x} \mid S \in \mathcal{W}\right\}$ is a Whitney stratification of $O_{x}$. This last statement will in turn be proved by exhibiting, for each $x \in \mathcal{M}$, a local chart in which we can apply Lemma 4.10.

Fix $x \in \mathcal{M}:=\mathrm{S}_{\rho}^{\circ}(f, \phi) \backslash \mathrm{S}(f, \phi)$. By Lemma 4.9 , there is a trivializing neighborhood $O_{x}$ with trivializing coordinates $(z, u)$. By shrinking $O_{x}$ if necessary, we can assume that $O_{x} \subseteq \mathcal{M}$. Let $B:=B(\bar{u}, \varepsilon) \subseteq \mathbb{R}^{S}$ denote the open ball defined in Lemma 4.9. In the coordinates $(z, u)$, we are in the situation of Lemma 4.10 when we take

$$
S=\left\{i \in\{1, \ldots q\}|| g_{i}(x) \mid \leq \rho\right\}, I:=E \cap S, J:=P \cap S .
$$


Since the assertion is trivial if $S$ is empty, we assume $S \neq \varnothing$. Applying Lemma 4.10, we obtain the Whitney stratification $\left\{\sigma_{K, L} \mid \sigma_{K, L} \neq \varnothing\right\}$ of $\Omega$, which induces the Whitney stratification $\left\{\sigma_{K, L} \cap B \mid \sigma_{K, L} \cap B \neq \varnothing\right\}$ of the open ball $B$, by Proposition 4.3(R). This in turn induces the product Whitney stratification $\left\{\mathcal{Z}_{x} \times\left(\sigma_{K, L} \cap B\right) \mid \sigma_{K, L} \cap B \neq \varnothing\right\}$ of $\mathcal{Z}_{x} \times B \simeq O_{x}$ by Proposition 4.3(P). We now note that each $\mathcal{Z}_{x} \times\left(\sigma_{K, L} \cap B\right)$ corresponds to $S_{K, L} \cap O_{x}$ in the local coordinates and hence, $\mathcal{W}$ is a Whitney stratification of $\mathcal{M}$ as claimed.

Using Lemma 4.10 in the corresponding local coordinates, we deduce easily the assertions (1) and (2) of Proposition 4.5 from the corresponding parts of Lemma 4.10, as well as the fact that $\alpha\left(\overline{S_{K, L}}\right)=\alpha\left(S_{K, L}\right)$ from its part (3).

It remains to prove the third assertion, that claims that $\alpha\left(S_{K, L}\right)=(0, \rho)$. Note first that the inclusion $\alpha\left(S_{K, L}\right) \subseteq(0, \rho)$ follows from the definitions of $\mathcal{M}$ and $\alpha$. The image $\alpha\left(S_{K, L}\right)$ is open since, by part $(2), \alpha_{\mid S_{K, L}}$ is a submersion. We show now that $\alpha\left(S_{K, L}\right)$ is also closed in $(0, \rho)$. By the connectedness of the interval $(0, \rho)$, and since $S_{K, L}$ is nonempty, this will imply $\alpha\left(S_{K, L}\right)=(0, \rho)$.

So consider a sequence $\left\{p_{n}\right\}$ in $S_{K, L}$ such that $\left\{\alpha\left(p_{n}\right)\right\}$ converges to $\alpha_{\infty} \in(0, \rho)$. By passing to a subsequence, we may assume that $\left\{p_{n}\right\}$ converges to some point $p_{\infty}$ in $\mathbb{S}^{n}$. Then $p_{\infty} \in \mathcal{M}$ as $\alpha\left(p_{\infty}\right)=\alpha_{\infty} \in(0, \rho)$. Therefore $\alpha_{\infty} \in \alpha\left(S_{K, L}\right)$, since we already know that $\alpha\left(\overline{S_{K, L}}\right)=\alpha\left(S_{K, L}\right)$. We have shown that $\alpha\left(S_{K, L}\right)$ is indeed closed in $(0, \rho)$ and the proof is complete.

Proof of Lemma 4.10. We already verified that $\left\{\sigma_{K, L} \mid \sigma_{K, L} \neq \varnothing\right\}$ is a partition of $\mathbb{R}^{S}$. We will use equations describing the different strata and their closures. Let $U_{i}$ denote the variable corresponding to the coordinate function $u \mapsto u_{i}$. The set $\sigma_{K, L}$ can be described by the expression

$$
\left(\vee_{i \in I}\left(U_{i} \neq 0\right)\right) \vee\left(\vee_{j \in J}\left(U_{j}<0\right)\right),
$$

ensuring that $u \in \Omega$, together with the (highly redundant) system

$$
\begin{cases}\left|U_{k}\right|>\left|U_{i}\right| & (k \in K, i \in I \backslash K) \\ \left|U_{\ell}\right|_{-}>\left|U_{j}\right|_{-} & (\ell \in L, j \in J \backslash L) \\ \left|U_{k}\right|=\left|U_{k^{\prime}}\right|^{\prime} & \left(k, k^{\prime} \in K\right) \\ \left|U_{\ell}\right|_{-}=\left|U_{\ell^{\prime}}\right|_{-} & \left(\ell, \ell^{\prime} \in L\right) \\ \left|U_{k}\right|=\left|U_{\ell}\right|_{-} & (k \in K, \ell \in L) .\end{cases}
$$

Let $\Omega_{K, L}$ denote the open subset of $\Omega$, described by (4.3), together with

$$
\begin{cases}\left|U_{k}\right|>\left|U_{i}\right| & (k \in K, i \in I \backslash K) \\ \left|U_{\ell}\right|_{-}>\left|U_{j}\right|_{-} & (\ell \in L, j \in J \backslash L) .\end{cases}
$$

We can obtain $\sigma_{K, L}$ as the intersection of $\Omega_{K, L}$ with the union, over all $\xi \in\{-1,1\}^{K}$, of the linear subspaces of $\mathbb{R}^{S}$ given by

$$
\begin{cases}\xi_{k} U_{k}=\xi_{k^{\prime}} U_{k^{\prime}} & \left(k, k^{\prime} \in K\right) \\ U_{\ell}=U_{\ell^{\prime}} & \left(\ell, \ell^{\prime} \in L\right) \\ \xi_{k} U_{k}=-U_{\ell} & (k \in K, \ell \in L) .\end{cases}
$$

Each of these linear subspaces has codimension $|K|+|L|-1$ since, in order to get a minimal system of equations, we only need to select a variable and, for each of the remaing $|K|+|L|-1$ variables, keep an equation determining its value. 
To prove that $\sigma_{K, L}$ is a smooth submanifold of $\mathbb{R}^{S}$, it suffices to show that any point $u$ lying in two of these linear subspaces necessarily lies outside of $\Omega$. Indeed, suppose $u$ is such a point. Then there exists $a \in K$ such that $u_{a}=-u_{a}$ and hence $u_{a}=0$. This implies $u_{i}=0$ for all $i \in I$ and then $u_{j}=0$ for all $j \in J$. Therefore $u \notin \Omega$. Thus $\sigma_{K, L}$ is indeed a locally closed smooth submanifold of $\mathbb{R}^{S}$. In particular, we have shown part (1) of Lemma 4.10.

For part (2), we observe that the restriction of $\alpha$ to each of the linear subspaces that make $\sigma_{K, L}$ agrees with either $\xi_{k} U_{k}$ for $k \in K$ or $-U_{\ell}$ for $\ell \in L$, which are non-zero linear maps on $\sigma_{K, L}$, as $\alpha$ does not take the value zero in $\Omega$.

We now claim that the following three conditions are equivalent:

$$
\begin{aligned}
\text { (i) } & \sigma_{K^{\prime}, L^{\prime}} \subseteq \overline{\sigma_{K, L}} \\
\text { (ii) } & \sigma_{K^{\prime}, L^{\prime}} \cap \overline{\sigma_{K, L}} \neq \varnothing \\
\text { (iii) } & K \subseteq K^{\prime} \text { and } L \subseteq L^{\prime} .
\end{aligned}
$$

To show this equivalence, we first observe that the closure $\overline{\sigma_{K, L}}$ in $\Omega$ is described by (4.3) together with the system obtained from (4.4) by replacing the strict inequalities by lax inequalities. This description shows the implication (iii) $\Rightarrow$ (i). The implication (i) $\Rightarrow$ (ii) is trivial. We show now (ii) $\Rightarrow$ (iii) by contraposition. Suppose $K \nsubseteq K^{\prime}$ and let $a \in K \backslash K^{\prime}$. As $a \in K$,

$$
\sigma_{K, L} \subseteq\left\{u|\alpha(u)=| u_{a} \mid\right\}
$$

which implies

$$
\overline{\sigma_{K, L}} \subseteq\left\{u|\alpha(u)=| u_{a} \mid\right\}
$$

Moreover, as $a \notin K^{\prime}$,

$$
\sigma_{K^{\prime}, L^{\prime}} \subseteq\left\{u|\alpha(u)>| u_{a} \mid\right\} .
$$

Thus $\overline{\sigma_{K, L}} \cap \sigma_{K^{\prime}, L^{\prime}}=\varnothing$. The case $L \nsubseteq L^{\prime}$ is shown in a similar way. So we have proved the equivalence of the three statements.

To prove part (3) it is enough to show that if $\sigma_{K^{\prime}, L^{\prime}} \subseteq \overline{\sigma_{K, L}}$, every point $u \in \sigma_{K^{\prime}, L^{\prime}}$ can be obtained as a limit of a sequence $\left\{u_{n}\right\}$ of points of $\sigma_{K, L}$ with the same value under $\alpha$. By the above characterization, $\sigma_{K^{\prime}, L^{\prime}} \subseteq \overline{\sigma_{K, L}}$ implies $K \subseteq K^{\prime}$ and $L \subseteq L^{\prime}$. This allows one to obtain the desired sequence to approach any point $u$ in $\sigma_{K^{\prime}, L^{\prime}}$ by slightly varying only the components $u_{t}$ with $t \in\left(K^{\prime} \backslash K\right) \cup\left(L^{\prime} \backslash L\right)$; for example, we may take $\left(u_{n}\right)_{t}:=(1-1 / n) u_{t}$, which is in $\sigma_{K, L}$ as it satisfies (4.3) and (4.4).

We finally show Whitney's condition $\mathrm{b}$, thus completing the proof that $\mathcal{W}$ is a Whitney stratification. The tangent space $\mathrm{T}_{x} \sigma_{K, L}$ at a point $u \in \sigma_{K, L}$ is the linear subspace given by

$$
\begin{cases}\operatorname{sgn}\left(u_{k}\right) U_{k}=\operatorname{sgn}\left(u_{k^{\prime}}\right) U_{k^{\prime}} & \left(k, k^{\prime} \in K\right) \\ U_{\ell}=U_{\ell^{\prime}} & \left(\ell, \ell^{\prime} \in L\right) \\ \operatorname{sgn}\left(u_{k}\right) U_{k}=-U_{\ell} & (k \in K, \ell \in L)\end{cases}
$$

where sgn $: \mathbb{R} \rightarrow\{-1,0,1\}$ is the sign function. Now assume $\sigma_{K^{\prime}, L^{\prime}} \cap \overline{\sigma_{K, L}} \neq \varnothing$ which, by (4.5), means that $K \subseteq K^{\prime}$ and $L \subseteq L^{\prime}$. Consider sequences of points $\left\{u_{n}\right\}$ and $\left\{u_{n}^{\prime}\right\}$ in $\sigma_{K, L}$ and $\sigma_{K^{\prime}, L^{\prime}}$, respectively, such that they both converge to $u \in \sigma_{K^{\prime}, L^{\prime}} \cap \overline{\sigma_{K, L}}$. By the definition of convergence we have that, for all $n$ large enough and $k \in K^{\prime}$,

$$
\operatorname{sgn}\left(u_{k}\right)=\operatorname{sgn}\left(\left(u_{n}\right)_{k}\right)=\operatorname{sgn}\left(\left(u_{n}^{\prime}\right)_{k}\right)
$$


as $u_{k} \neq 0$ for all $k \in K^{\prime}$. This implies that for all $n$ large enough, the line $\overline{u_{n} u_{n}^{\prime}}$ through $u_{n}$ and $u_{n}^{\prime}$ lies inside $\mathrm{T}_{u_{n}} \sigma_{K, L}$ as, by the equations (4.6) and the inclusions $K \subseteq K^{\prime}$ and $L \subseteq L^{\prime}$, both $u_{n}$ and $u_{n}^{\prime}$ lie in $\mathrm{T}_{u_{n}} \sigma_{K, L} \subseteq \mathrm{T}_{u_{n}^{\prime}} \sigma_{K^{\prime}, L^{\prime}}$. As this inclusion is preserved in the limit, we see that Whitney's condition b holds.

\section{Topology}

In this section, we introduce two tools to construct isomorphisms of homology groups: an Explicit Homological Nerve Theorem for Čech complexes and a Homological InclusionExclusion Transfer. These tools will combine topological information of basic semialgebraic sets to obtain such information for general semialgebraic sets.

\subsection{Explicit Homological Nerve Theorem}

Recall (from §2.4) the definition, for a finite set of points $\mathcal{X} \subseteq \mathbb{R}^{m}$ and $\varepsilon>0$, of the $\check{C}$ ech complex of $\mathcal{X}$ of radius $\varepsilon$. By the Nerve Theorem [40, Corollary 4G.3], the Cech complex $\check{\mathrm{C}}_{\mathcal{\varepsilon}}(\mathcal{X})$ is homotopically equivalent to the open $\varepsilon$-neighborhood $\mathcal{U}(\mathcal{X}, \varepsilon)$ around $\mathcal{X}$ defined in $§ 2.4$. In particular, $\mathcal{U}(\mathcal{X}, \varepsilon)$ and $\check{\mathrm{C}}_{\varepsilon}(\mathcal{X})$ have the same homology.

We next exhibit a map that realizes this isomorphism in homology.

Consider the free simplex with vertex set $\mathcal{X}$, which is defined as the set

$$
\Delta^{\mathcal{X}}:=\left\{\sum_{x \in \mathcal{X}} t_{x}[x] \mid \text { for all } x \in \mathcal{X}, t_{x} \geq 0, \sum_{x \in \mathcal{X}} t_{x}=1\right\} \subseteq \mathbb{R}^{\mathcal{X}}
$$

formed by the formal convex combinations of the points of $\mathcal{X}$. Here we use the notation $[x]$ to distinguish the vertex $[x]$ in $\Delta^{\mathcal{X}}$ from the point $x \in \mathcal{X} \subseteq \mathbb{R}^{m}$. For $\sigma \in \check{\mathrm{C}}_{\varepsilon}(\mathcal{X})$, the free simplex $\Delta^{\sigma}$ lies inside $\Delta^{\mathcal{X}}$ as a face and this correspondence is compatible with intersections in the sense that $\Delta^{\sigma \cap \sigma^{\prime}}=\Delta^{\sigma} \cap \Delta^{\sigma^{\prime}}$. This implies that by taking the union of all these faces, we get the following realization

$$
\left[\check{\mathrm{C}}_{\varepsilon}(\mathcal{X})\right]:=\bigcup\left\{\Delta^{\sigma} \mid \sigma \in \check{\mathrm{C}}_{\varepsilon}(\mathcal{X})\right\}
$$

of the abstract simplicial complex $\check{\mathrm{C}}_{\varepsilon}(\mathcal{X})$ inside $\Delta^{\mathcal{X}}$. In fact, this is the simplest geometric realization of the given abstract simplicial complex.

Consider the affine map $\pi: \mathbb{R}^{\mathcal{X}} \rightarrow \mathbb{R}^{m}$ that sends the vertex $[x]$ to the corresponding point $x$. In other words,

$$
\pi\left(\sum_{x \in \mathcal{X}} t_{x}[x]\right)=\sum_{x \in \mathcal{X}} t_{x} x .
$$

Clearly, $\pi$ maps the free simplex $\Delta^{\mathcal{X}}$ onto the the convex hull $\operatorname{conv}(\mathcal{X})$ of $\mathcal{X}$ in $\mathbb{R}^{m}$. The next lemma implies that $\pi$ maps the realization $\left[\check{\mathrm{C}}_{\varepsilon}(\mathcal{X})\right]$ to $\mathcal{U}(\mathcal{X}, \varepsilon)$.

Lemma 5.1. Let $\varepsilon>0$ and $\mathcal{X} \subseteq \mathbb{R}^{m}$ be a finite family of points. If $\bigcap_{x \in \mathcal{X}} B(x, \varepsilon) \neq \varnothing$, then $\operatorname{conv}(\mathcal{X}) \subseteq \mathcal{U}(\mathcal{X}, \varepsilon)$.

Proof. Without loss of generality, by Carathéodory's Theorem [66, Proposition 1.15], we can assume that $\operatorname{conv}(\mathcal{X})$ is a simplex. Suppose $\bigcap_{x \in \mathcal{X}} B(x, \varepsilon) \neq \varnothing$. For a nonempty $\sigma \subseteq \mathcal{X}$, take $p^{\prime} \in \bigcap_{x \in \sigma} B(x, \varepsilon)$, and let $p_{\sigma}$ be the closest point to $p^{\prime}$ in $\operatorname{conv}(\sigma)$. Then $p_{\sigma} \in$ $\bigcap_{x \in \sigma} B(x, \varepsilon)$. By perturbing, we can assume that $p_{\sigma}$ lies in the relative interior of $\operatorname{conv}(\sigma)$. 
We now consider the barycentric subdivision of $\operatorname{conv}(\mathcal{X})$ with respect to the family of points $\left\{p_{\sigma} \mid \sigma \subseteq \mathcal{X}\right\}$, which is a barycentric subdivision where we take $p_{\sigma}$ instead of taking the centroid in the relative interior of each face $\sigma \subseteq \mathcal{X}$. It is sufficient to show that conv $(\Delta) \subseteq \mathcal{U}(\mathcal{X}, \varepsilon)$ for every maximal simplex of this subdivision. Every such simplex $\Delta$ has the form $\operatorname{conv}\left(p_{\left\{x_{1}\right\}}, p_{\left\{x_{1}, x_{2}\right\}}, \ldots, p_{\mathcal{X}}\right)$, where $x_{i} \in \mathcal{X}$, so we have $p_{\left\{x_{1}, \ldots, x_{a}\right\}} \in \bigcap_{i=1}^{a} B\left(x_{i}, \varepsilon\right) \subseteq B\left(x_{1}, \varepsilon\right)$ for each of each of its vertices $p_{\left\{x_{1}, \ldots, x_{a}\right\}}$. Therefore, $\Delta \subseteq B\left(x_{1}, \varepsilon\right) \subseteq \mathcal{U}(\mathcal{X}, \varepsilon)$ by convexity.

We can now state the Explicit Homological Nerve Theorem for Čech complexes.

Theorem 5.2 (Explicit Homological Nerve Theorem). The restriction $\tilde{\pi}:\left[\check{C}_{\varepsilon}(\mathcal{X})\right] \rightarrow$ $\mathcal{U}(\mathcal{X}, \varepsilon)$ of the affine map $\pi$ induces an isomorphism in homology:

$$
\tilde{\pi}_{*}: H_{*}\left(\left[\check{C}_{\varepsilon}(\mathcal{X})\right]\right) \rightarrow H_{*}(\mathcal{U}(\mathcal{X}, \varepsilon)) .
$$

Proof. Let $\sigma \in \check{\mathrm{C}}_{\varepsilon}(\mathcal{X})$. Then $\bigcap_{x \in \mathcal{X}} B(x, \varepsilon) \neq \varnothing$ and so, by Lemma 5.1 applied to $\sigma$, $\operatorname{conv}(\sigma) \subseteq \mathcal{U}(\sigma, \varepsilon) \subseteq \mathcal{U}(\mathcal{X}, \varepsilon)$. As $\left[\check{\mathrm{C}}_{\varepsilon}(\mathcal{X})\right]=\bigcup_{\sigma \in \check{\mathrm{C}}_{\varepsilon}(\mathcal{X})} \Delta^{\sigma}$ and $\operatorname{conv}(\sigma)=\pi\left(\Delta^{\sigma}\right)$, it follows that $\pi\left(\left[\check{\mathrm{C}}_{\varepsilon}(\mathcal{X})\right]\right) \subseteq \mathcal{U}(\mathcal{X}, \varepsilon)$. Thus $\pi$ is a continuous map $\left[\check{\mathrm{C}}_{\varepsilon}(\mathcal{X})\right] \rightarrow \mathcal{U}(\mathcal{X}, \varepsilon)$. It only remains to prove that it induces an isomorphism in homology.

Let $\left\{\phi_{x}\right\}_{x \in \mathcal{X}}$ be a partition of unity in $\mathcal{U}(\mathcal{X}, \varepsilon)$ subordinate to $\{B(x, \varepsilon)\}_{x \in \mathcal{X}}$. That is, the $\phi_{x}$ are continuous maps $\phi_{x}: \mathcal{U}(\mathcal{X}, \varepsilon) \rightarrow[0,1]$ such that $\phi_{x}$ is zero outside $B(x, \varepsilon)$ and $\sum_{x \in \mathcal{X}} \phi_{x}=1$. (For example, we could take $\phi_{x}:=\frac{\rho_{x}}{\sum_{x \in \mathcal{X}} \rho_{x}}$ with $\rho_{x}(p):=\max \{\varepsilon-\| p-$ $x \|, 0\}$.) We define the continuous map

$$
\varphi: \mathcal{U}(\mathcal{X}, \varepsilon) \rightarrow\left[\check{\mathrm{C}}_{\mathcal{\varepsilon}}(\mathcal{X})\right], p \mapsto \sum_{x \in \mathcal{X}} \phi_{x}(p)[x]
$$

and will show that $\pi \circ \varphi$ is homotopic to the identity $\operatorname{id}_{\mathcal{U}(\mathcal{X}, \varepsilon)}$. To do so, consider the linear homotopy

$$
t \mapsto t(\pi \circ \varphi)+(1-t) \operatorname{id}_{\mathcal{U}(\mathcal{X}, \varepsilon)}
$$

between $\pi \circ \varphi$ and $\operatorname{id}_{\mathcal{U}(\mathcal{X}, \varepsilon)}$. To show that this linear homotopy restricts to a homotopy of functions $\mathcal{U}(\mathcal{X}, \varepsilon) \rightarrow \mathcal{U}(\mathcal{X}, \varepsilon)$, we only have to check that for every $p \in \mathcal{U}(x, \varepsilon)$, the segment $[\pi(\varphi(p)), p]$ is contained in $\mathcal{U}(x, \varepsilon)$.

In order to check this, put $\mathcal{X}:=\left\{x \in \mathcal{X} \mid \phi_{x}(p) \neq 0\right\}$ and note that

$$
\pi(\varphi(p))=\sum_{x \in \mathcal{X}} \phi_{x}(p) x \in \operatorname{conv}(\mathcal{X})
$$

We have $p \in \bigcap_{x \in \mathcal{X}} B(x, \varepsilon)$ since $\phi_{x}(p) \neq 0$ implies $d(x, p)<\varepsilon$. By Lemma 5.1 we have $\operatorname{conv}(\mathcal{X}) \subseteq \mathcal{U}(\mathcal{X}, \varepsilon)$. So $\pi(\varphi(p)) \in \mathcal{U}(\mathcal{X}, \varepsilon)$. Hence there exists $\tilde{x} \in \mathcal{X}$ such that $\pi(\varphi(p)) \in B(\tilde{x}, \varepsilon)$. Since also $p \in B(\tilde{x}, \varepsilon)$, we have $[p, \pi(\varphi(p))] \subseteq B(\tilde{x}, \varepsilon) \subseteq \mathcal{U}(\mathcal{X}, \varepsilon)$.

So we have shown that $\pi \circ \varphi$ is homotopic to the identity. Therefore, $\pi_{*}: H_{\ell}\left(\left[\check{\mathrm{C}}_{\varepsilon}(\mathcal{X})\right]\right) \rightarrow$ $H_{\ell}(\mathcal{U}(\mathcal{X}, \varepsilon))$ is an epimorphism for every $\ell$. Now, by the Nerve Theorem [40, Corollary $4 \mathrm{G} .3], H_{\ell}\left(\left[\check{\mathrm{C}}_{\varepsilon}(\mathcal{X})\right]\right)$ and $H_{\ell}(\mathcal{U}(\mathcal{X}, \varepsilon))$ are isomorphic finitely generated abelian groups. We conclude that $\pi$ induces an isomorphism in homology, because a surjective homomorphism between isomorphic finitely generated abelian groups is an isomorphism [50, Exercises 4.2(10)].

Remark 5.3. Theorem 5.4 below gives an alternative way of proving Theorem 5.2 without using the Nerve Theorem. 


\subsection{Homological Inclusion-Exclusion Transfer}

The title of the subsection refers to the idea of inferring information on the homology of a space $X$ (or a map between spaces) from the homology of intersections of subspaces, akin to the combinatorial inclusion-exclusion principle.

Let $X$ be a topological space and $C \cdot(X)$ be its singular chain complex. For $A, B \subseteq X$ we denote by $C_{\bullet}(A+B)$ the subcomplex of $C_{\bullet}(A \cup B)$ generated by the singular simplices that either lie inside $A$ or inside $B$. We will say that a finite family $\left\{X_{i}\right\}_{i \in I}$ of subsets of $X$ satisfies the Mayer-Vietoris hypothesis when, for every non-empty $J \subseteq I$ and $k \in I \backslash J$, the inclusion of chain complexes

$$
C \bullet\left(X_{k}+\bigcup_{j \in J} X_{j}\right) \hookrightarrow C \bullet\left(X_{k} \cup \bigcup_{j \in J} X_{j}\right),
$$

induces an isomorphism in homology. We will say that it satisfies the inductive MayerVietoris hypothesis when, for all finite families $\left\{F_{\ell}\right\}_{\ell \in L}$ of subsets of $I$, the family of intersections $\left\{\cap_{h \in F_{\ell}} X_{h}\right\}_{\ell \in L}$ satisfies the Mayer-Vietoris hypothesis.

The reason to introduce this last notion is that it gives a common name to the three main situations that we will encounter and in which this condition holds:

1) The family $\left\{X_{i}\right\}_{i \in I}$ is a family of open subsets of $\bigcup_{i \in I} X_{i}$. The inductive Mayer-Vietoris hypothesis holds due to [40, Proposition 2.21].

2) The family $\left\{X_{i}\right\}_{i \in I}$ is a family of closed subcomplexes of a CW-complex. The inductive Mayer-Vietoris hypothesis holds due to [51, Cor. 8.44].

3) The family $\left\{X_{i}\right\}_{i \in I}$ is a family of closed semialgebraic sets in $\mathbb{R}^{N}$. The inductive MayerVietoris hypothesis holds due to the Semialgebraic Triangulation Theorem [9, Theorem 9.2.1] combined with situation 2) above.

In all these three situations, the inductive Mayer-Vietoris hypothesis will allow us to use the Mayer-Vietoris exact sequence in inductive arguments, such as the one for the following theorem.

Theorem 5.4 (Homological Inclusion-Exclusion Transfer). Let $X$ and $Y$ be topological spaces and $\left\{X_{i}\right\}_{i \in I},\left\{Y_{i}\right\}_{i \in I}$ be finite families of subsets of $X$ and $Y$, respectively, satisfying the inductive Mayer-Vietoris hypothesis. We assume that $X=\bigcup_{i \in I} X_{i}$ and $Y=\bigcup_{i \in I} Y_{i}$. Moreover, let $f: X \rightarrow Y$ be a continuous map such that $f\left(X_{i}\right) \subseteq Y_{i}$ for all $i \in I$. Let $k$ be an integer such that for all nonempty $J \subseteq I$ with $|J| \leq k$, the morphism

$$
H_{\ell}(f): H_{\ell}\left(\cap_{j \in J} X_{j}\right) \rightarrow H_{\ell}\left(\cap_{j \in J} Y_{j}\right)
$$

is an isomorphism for $\ell<k$ and an epimorphism for $\ell=k$. Then

$$
H_{\ell}(f): H_{\ell}(X) \rightarrow H_{\ell}(Y)
$$

is an isomorphism for $\ell<k$ and an epimorphism for $\ell=k$.

The following is an immediate consequence of Theorem 5.4.

Corollary 5.5. Under the assumptions of Theorem 5.4 if, for all nonempty $J \subseteq I$, $f: \cap_{j \in J} X_{j} \rightarrow \cap_{j \in J} Y_{j}$ induces an isomorphism in homology, then $f: X \rightarrow Y$ induces an isomorphism in homology. 
Proof of Theorem 5.4. The proof is by induction on the size of $I$, for arbitrary $k$. The assertion is trivial when $I$ is a singleton.

Let $I=I^{\prime} \cup\left\{i_{0}\right\}$ with $i_{0} \notin I^{\prime}$. By assumption, we have $f\left(\cup_{i \in I^{\prime}} X_{i}\right) \subseteq \cup_{i \in I^{\prime}} Y_{i}, f\left(X_{i_{0}}\right) \subseteq$ $Y_{i_{0}}$ and $f\left(\cup_{i \in I^{\prime}}\left(X_{i_{0}} \cap X_{i}\right)\right) \subseteq \cup_{i \in I^{\prime}}\left(Y_{i_{0}} \cap Y_{i}\right)$. By induction hypothesis, the maps

$$
\beta_{\ell}^{1}: H_{\ell}\left(X_{i_{0}}\right) \rightarrow H_{\ell}\left(Y_{i_{0}}\right) \text { and } \beta_{\ell}^{2}: H_{\ell}\left(\cup_{i \in I^{\prime}} X_{i}\right) \rightarrow H_{\ell}\left(\cup_{i \in I^{\prime}} Y_{i}\right)
$$

induced by $f$ are isomorphisms for $\ell<k$ and epimorphisms for $\ell=k$, and the maps

$$
\alpha_{\ell}: H_{\ell}\left(\cup_{i \in I^{\prime}}\left(X_{i_{0}} \cap X_{i}\right)\right) \subseteq H_{\ell}\left(\cup_{i \in I^{\prime}}\left(Y_{i_{0}} \cap Y_{i}\right)\right)
$$

are isomorphisms for $\ell<k-1$ and epimorphisms for $\ell=k-1$. Here we view $\cap_{j \in J}\left(X_{i_{0}} \cap\right.$ $\left.X_{j}\right)=X_{i_{0}} \cap\left(\cap_{j \in J} X_{j}\right)$ as an intersection of $|J|+1$ subsets, for $J \subseteq I^{\prime}$ with $|J| \leq k-1$. (Note that the inductive Mayer-Vietoris hypothesis is necessary to apply the induction step, as it guarantees that the families $\left\{X_{i_{0}} \cap X_{j}\right\}_{j \in J}$ and $\left\{Y_{i_{0}} \cap Y_{j}\right\}_{j \in J}$ satisfy the induction hypothesis; this is not necessarily the case with the Mayer-Vietoris hypothesis.)

The map of pairs $f:\left(\cup_{i \in I^{\prime}} X_{i}, X_{i_{0}}\right) \rightarrow\left(\cup_{i \in I^{\prime}} Y_{i}, Y_{i_{0}}\right)$, and the fact that these pairs satisfy the Mayer-Vietoris hypothesis, induce the commutative diagram of Mayer-Vietoris sequences shown in Figure 1, where $\alpha_{\ell}, \beta_{\ell}$ and $\gamma_{\ell}$ are the maps in homology induced by $f$.

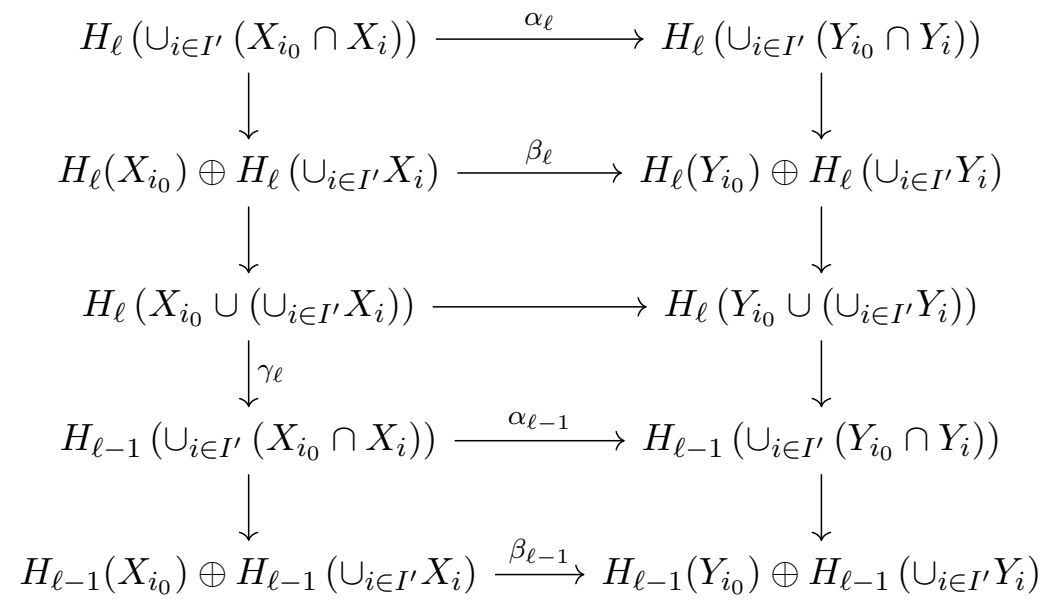

Figure 1 Natural maps of Mayer-Vietoris sequences in the proof of Theorem 5.4.

In this figure, the induction hypothesis ensures that $\alpha_{\ell}$ is an isomorphism for $\ell<k-1$, an epimorphism for $\ell=k-1$, and that $\beta_{\ell}$ is an isomorphism for $\ell<k$ and an epimorphism for $\ell=k$. This gives us two cases to consider: $\ell \leq k-1$ and $\ell=k$.

If $\ell \leq k-1$, then $\alpha_{\ell-1}, \beta_{\ell-1}$ and $\beta_{\ell}$ are isomorphisms and $\alpha_{\ell}$ is an epimorphism. Therefore, by the Five Lemma [52, Proposition 2.72(iii)], $\gamma_{\ell}$ is an isomorphism.

Otherwise, if $\ell=k$, then $\beta_{\ell}$ and $\alpha_{\ell-1}$ are epimorphisms, and $\beta_{\ell-1}$ is an isomorphism. Therefore, by the Four Lemma [52, Proposition 2.72(i)], $\gamma_{\ell}$ is an epimorphism.

The statement now follows by induction.

Remark 5.6. Theorem 5.4 can be considered a homological version of the Vietoris-Begle Theorem [61, p. 344] for homology in terms of coverings. For example, one can see that for a locally trivial fibration $\pi: E \rightarrow B$ with $(k-1)$-connected fiber $F$, the homological inclusion-exclusion transfer implies the homological Vietoris-Begle Theorem since, for every trivializing open subset $U \subseteq B, H_{\ell}(F \times U) \rightarrow H_{\ell}(U)$ is an isomorphism for $\ell<k$ and an epimorphism for $\ell=k$, 


\section{Algorithms}

Our algorithm follows the steps described (with broad strokes) in Section 2:

(1) We estimate the intersection condition $\bar{\kappa}(f)$. We do this in Algorithm $\bar{\kappa}$-Estimate in Subsection 6.2.

(2) We construct clouds of points $\mathcal{X}_{j}^{\alpha_{j}}$ approximating the atomic sets $S_{j}^{\alpha_{j}}$, for $j \in$ $\{1, \ldots, q\}$ and $\propto_{j} \in\{\leq,=, \geq\}$, and satisfying that intersections of clouds approximate the corresponding intersections of sets. We use these clouds and our estimate on $\bar{\kappa}(f)$ to produce a simplicial complex $\mathfrak{C}$ having the same homology as $\mathbf{S}(f, \Phi)$. This is Algorithm Simplicial in Subsection 6.3.

(3) We computation the homology of $\mathfrak{C}$. This is standard. But we recall the procedure and its complexity in Subsection 6.4.

To do these computations, a sequence of grids on $\mathbb{S}^{n}$ is necessary. In this section we first describe the nature of these grids (and how to construct them) and then proceed to describe and analyse the complexity of the algorithms in the steps above. This complexity analysis is condition-based: the bounds are in terms of $\bar{\kappa}(f)$, in addition to the general parameters $n, q$ and $D$.

\subsection{Grids}

The algorithm uses a sequence of grids on the sphere, both for estimating $\bar{\kappa}(f)$ and for sampling points on $\mathbb{S}^{n}$. These grids are simply constructed by projection onto the unit sphere of a uniform grid in the boundary of a cube. This sequence of grids has been used in $[30,26,29,15]$ and its basic properties have been proved in these papers. We will therefore be concise in what follows.

For $\ell \in \mathbb{N}$, let $\mathcal{G}_{\ell}$ be the image on $\mathbb{S}^{n}$ under the projection $x \rightarrow \frac{x}{\|x\|}$ of the set of points $x \in \mathbb{Z}^{n+1}$ with $\|x\|_{\infty}=\left\lceil 2^{\ell} \sqrt{n}\right\rceil$. Further, let $r_{\ell}:=2^{-\ell}$. Then,

$$
\left|\mathcal{G}_{\ell}\right|=\left(n 2^{\ell}\right)^{\mathcal{O}(n)}
$$

and

$$
\mathbb{S}^{n} \subseteq \bigcup_{x \in \mathcal{G}_{\ell}} B_{\mathbb{S}}\left(x, r_{\ell}\right) \subseteq \bigcup_{x \in \mathcal{G}_{\ell}} B\left(x, r_{\ell}\right)
$$

Note that the last implies that $d_{H}\left(\mathcal{G}_{\ell}, \mathbb{S}^{n}\right) \leq r_{\ell}$. We finally observe that, given $\ell$, the grid $\mathcal{G}_{\ell}$ is easily computable.

\subsection{Estimating the condition}

Recall the Definition 3.2 of the real homogeneous condition number $\kappa(f)$ of $f \in \mathcal{H}_{\boldsymbol{d}}[q]$. We use the Lipschitz character of the inverse of $\kappa$ as a map on the sphere to estimate global bounds for $\kappa$ based on a finite number of point evaluations.

Lemma 6.1. Let $f \in \mathcal{H}_{\boldsymbol{d}}[q], \ell \in \mathbb{N}$, and

$$
\kappa_{\ell}(f):=\max \left\{\kappa(f, x) \mid x \in \mathcal{G}_{\ell}\right\} .
$$

Then $\kappa_{\ell}(f) \leq \kappa(f)$. Moreover, if $2 D \kappa_{\ell}(f) r_{\ell}<1$, we have

$$
\kappa(f) \leq \frac{\kappa_{\ell}(f)}{1-2 D \kappa_{\ell}(f) r_{\ell}} .
$$


Proof. The first claimed inequality is trivial. To prove the second we recall that, by [15, Proposition 4.7], the map $\mathbb{S}^{n} \rightarrow[0,1], x \mapsto \kappa(f, x)^{-1}$ is $D$-Lipschitz continuous with respect to the Riemannian distance on $\mathbb{S}^{n}$, and so $2 D$-Lipschitz with respect to the Euclidean distance on $\mathbb{S}^{n}$. Let $x_{*} \in \mathbb{S}^{n}$ be such that $\kappa(f)=\kappa\left(f, x_{*}\right)$. By the inclusions (6.2), there exists $x \in \mathcal{G}_{\ell}$ such that $d\left(x, x_{*}\right)<r_{\ell}$. Using the Lipschitz property for the pair $\left(x, x_{*}\right)$ it follows that

$$
\frac{1}{\kappa_{\ell}(f)}-2 D r_{\ell} \leq \frac{1}{\kappa(f, x)}-2 D r_{\ell} \leq \frac{1}{\kappa\left(f, x_{*}\right)}=\frac{1}{\kappa(f)}
$$

The desired inequality follows.

We immediately derive analogous bounds for the real homogeneous intersection condition number.

Corollary 6.2. Let $f \in \mathcal{H}_{\boldsymbol{d}}[q], \ell \in \mathbb{N}$, and

$$
\bar{\kappa}_{\ell}(f):=\max \left\{\kappa_{\ell}\left(f^{L}\right)|L \subseteq\{1, \ldots, q\},| L \mid \leq n+1\right\} .
$$

Then $\bar{\kappa}_{\ell}(f) \leq \bar{\kappa}(f)$. Moreover, if $2 D \bar{\kappa}_{\ell}(f) r_{\ell}<1$, we have

$$
\bar{\kappa}(f) \leq \frac{\bar{\kappa}_{\ell}(f)}{1-2 D \bar{\kappa}_{\ell}(f) r_{\ell}} .
$$

Corollary 6.2 motivates (and provides a proof of correctness for) the following algorithm.

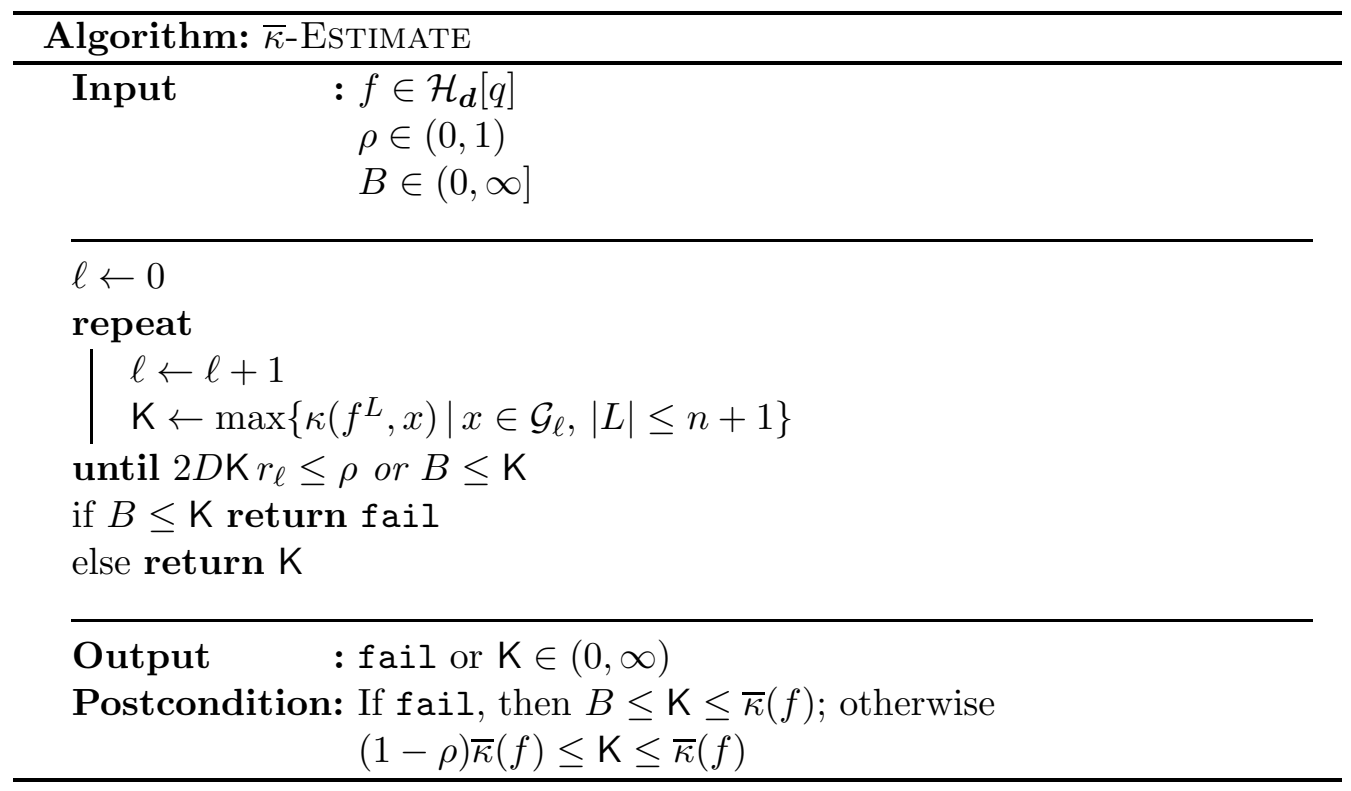

Proposition 6.3. Algorithm $\bar{\kappa}$-ESTIMATE is correct. Its cost on input $(f, \rho, B)$ is bounded by

$$
\left(q n D \min \{B, \bar{\kappa}(f)\} \rho^{-1}\right)^{\mathcal{O}(n)} .
$$

Proof. The correctness follows from Corollary 6.2 and the stopping criterion, noting that at each iteration we have $\mathrm{K}=\bar{\kappa}_{\ell}(f) \leq \bar{\kappa}(f)$.

To prove the cost bound assume that, after $\ell$ iterations, we have

$$
\ell \geq \log _{2}\left(2 D \mathscr{K} \rho^{-1}\right),
$$


where $\mathscr{K}:=\min \{B, \mathrm{~K}\}$. Then $r_{\ell}=2^{-\ell} \leq \frac{\rho}{D \mathscr{K}}$. If $B>\mathrm{K}$ then $\mathscr{K}=\mathrm{K}, r_{\ell} \leq \frac{\rho}{2 D \mathrm{~K}}$, and the algorithm halts. On the other hand, if $B \leq \mathrm{K}$, the algorithm halts as well. Thus we have shown that the algorithms halts after at most

$$
T:=\log _{2}\left(2 D \min \{B, \bar{\kappa}(f)\} \rho^{-1}\right)
$$

iterations. At the $\ell$ th iteration, where $\ell \leq T$, the number of points in $\mathcal{G}_{\ell}$ is, by (6.1), bounded by

$$
\left(n 2^{\ell}\right)^{\mathcal{O}(n)}=\left(n 2^{T}\right)^{\mathcal{O}(n)} .
$$

For each point $x \in \mathcal{G}_{\ell}$ we compute the value of $\kappa\left(f^{L}, x\right)$ for at $\operatorname{most} \sum_{i=1}^{\min \{q, n+1\}}\left(\begin{array}{c}q \\ i\end{array}\right) \leq$ $(q+1)^{n+1}$ subsets $L$. And each of these computations can be done in $\mathcal{O}\left(N+n^{3}\right)$ operations (see $[45, \S 2.5]$ ) where we recall that $N=\operatorname{dim} \mathcal{H}_{\boldsymbol{d}}[q]$. (Actually, we compute $\kappa$ up to a factor of $\sqrt{n}$, but we will disregard this fact for simplicity.) It is easy to see that

$$
N \leq(n D)^{\mathcal{O}(n)},
$$

from where it follows that each $\kappa\left(f^{L}, x\right)$ is computed with cost $(n D)^{\mathcal{O}(n)}$.

Putting all the previous bounds together we bound the cost of the computation by

$$
T\left(n 2^{T}\right)^{\mathcal{O}(n)}(q+1)^{n+1}(n D)^{\mathcal{O}(n)} \leq\left(q n D \min \{B, \bar{\kappa}(f)\} \rho^{-1}\right)^{\mathcal{O}(n)},
$$

which finishes the proof.

Remark 6.4. Algorithm $\bar{\kappa}$-Estimate estimates $\bar{\kappa}(f)$ up to a precision $\rho$ in finite time, provided this condition number is not too large (not much bigger than $B$ ). When $B=\infty$ is given as input, it estimates $\bar{\kappa}(f)$ up to this precision but its running time is not bounded. In particular, if $\bar{\kappa}(f)=\infty$, then the algorithm loops forever.

Proof of Proposition 2.2. This is just a particular case of Proposition 6.3 with $B=\infty$ and $\rho=0.01$.

\subsection{Computation of simplicial complexes}

Given $f \in \mathcal{H}_{\boldsymbol{d}}[q]$, a lax formula $\Phi$, and $\ell \in \mathbb{N}$, we define the finite cloud of points

$$
\mathcal{X}_{\ell}(f, \Phi):=\mathrm{S}_{D^{1 / 2} r_{\ell}}^{\circ}(f, \Phi) \cap \mathcal{G}_{\ell} .
$$

In the special case of atomic formulas $f_{j} \propto_{j} 0$, we will write $\mathcal{X}_{j}^{\propto_{j}}$ for the corresponding cloud. The following theorem gives sufficient conditions on $\ell$ and $\bar{\kappa}(f)$ for the clouds $\mathcal{X}_{\ell}(f, \Phi)$ to approximate, as in the hypothesis of Theorem 2.4, the sets $\mathrm{S}(f, \Phi)$.

Theorem 6.5 (Sampling Theorem). Assume $f \in \mathcal{H}_{\boldsymbol{d}}[q]$ and $\ell \in \mathbb{N}$ are such that $13 D^{2} \bar{\kappa}(f)^{2} r_{\ell}<1$. Then, for every lax formula $\Phi$, we have

$$
d_{H}\left(\mathcal{X}_{\ell}(f, \Phi), \mathrm{S}(f, \Phi)\right)<3 D^{1 / 2} \bar{\kappa}(f) r_{\ell} .
$$

Proof. Without loss of generality we can assume that $\Phi$ is in DNF, as this assumption does not change the underlying set. Furthermore, we can assume that $\Phi$ is basic due to the inequality

$$
d_{H}\left(\cup_{i=1}^{t} A_{i}, \cup_{i=1}^{t} B_{i}\right) \leq \max _{i} d_{H}\left(A_{i}, B_{i}\right)
$$


of the Hausdorff distance and the fact that $\mathcal{X}_{\ell}\left(f, \Psi_{0} \vee \Psi_{1}\right)=\mathcal{X}_{\ell}\left(f, \Psi_{0}\right) \cup \mathcal{X}_{\ell}\left(f, \Psi_{1}\right)$.

By the construction of $\mathcal{X}_{\ell}$,

$$
\mathcal{X}_{\ell}(f, \Phi) \subseteq \mathrm{S}_{D^{1 / 2} r_{\ell}}^{\circ}(f, \Phi) \subseteq \mathcal{U}\left(\mathrm{S}(f, \Phi), 3 D^{1 / 2} \bar{\kappa}(f) r_{\ell}\right),
$$

the last by Proposition 2.6 and (2.2). By (6.2), for all $x \in \mathrm{S}(f, \Phi)$, there is some $g_{x} \in \mathcal{G}_{\ell}$ such that $d_{\mathbb{S}}\left(x, g_{x}\right) \leq d\left(x, g_{x}\right)<r_{\ell}$. Thus, by $(2.4), g_{x} \in \mathcal{U}_{\mathbb{S}}\left(\mathrm{S}(f, \Phi), r_{\ell}\right) \subseteq \mathrm{S}_{D^{1 / 2} r_{\ell}}^{\circ}(f, \Phi)$ and so $g_{x} \in \mathcal{X}_{\ell}(f, \Phi)$. Hence

$$
\mathrm{S}(f, \Phi) \subseteq \mathcal{U}\left(\mathcal{X}_{\ell}(f, \Phi), r_{\ell}\right) \subseteq \mathcal{U}\left(\mathcal{X}_{\ell}(f, \Phi), 3 D^{1 / 2} \bar{\kappa}(f) r_{\ell}\right),
$$

as $D \geq 1$ and $\bar{\kappa}(f) \geq 1$. The inequality on the Hausdorff distance follows from the two inclusions above.

We can now put together the Homology Witness Theorem 2.4 and the Sampling Theorem 6.5. The fundamental observation to make is that one only needs to sample from each of the $3 q$ atomic sets associated with $f \in \mathcal{H}_{\boldsymbol{d}}[q]$ defined in (2.3). The following (trivial) identity

$$
\mathcal{X}_{\ell}(f, \Phi)=\Phi\left(\mathcal{X}_{1}^{\leq}, \mathcal{X}_{1}^{=}, \mathcal{X}_{1}^{\geq}, \ldots, \mathcal{X}_{q}^{\leq}, \mathcal{X}_{q}^{=}, \mathcal{X}_{q}^{\geq}\right)
$$

allows us to obtain, for any lax formula $\Phi$, the cloud $\mathcal{X}_{\ell}(f, \Phi)$ by sampling from these atomic sets.

Proposition 6.6. Let $f \in \mathcal{H}_{\boldsymbol{d}}[q], \varepsilon>0$, and $\ell \in \mathbb{N}$ be such that

$$
9 D^{1 / 2} \bar{\kappa}(f) r_{\ell}<\varepsilon<\frac{1}{14 D^{3 / 2} \bar{\kappa}(f)} .
$$

Then, for all lax formulas $\Phi$ over $f$, the semialgebraic set $\mathrm{S}(f, \Phi) \subseteq \mathbb{S}^{n}$ and the simplicial complex

$$
\mathfrak{C}:=\Phi\left(\check{C}_{\varepsilon}\left(\mathcal{X}_{1}^{\leq}\right), \check{C}_{\varepsilon}\left(\mathcal{X}_{1}^{=}\right), \check{C}_{\varepsilon}\left(\mathcal{X}_{1}^{\geq}\right), \ldots, \check{C}_{\varepsilon}\left(\mathcal{X}_{q}^{\leq}\right), \check{C}_{\varepsilon}\left(\mathcal{X}_{q}^{=}\right), \check{C}_{\varepsilon}\left(\mathcal{X}_{q}^{\geq}\right)\right)
$$

have the same homology.

Proof. This follows from the Homology Witness Theorem 2.4 applied to $f \in \mathcal{H}_{\boldsymbol{d}}[q]$ and to the finite sets $\mathcal{X}_{j}^{\propto_{j}}$ associated to the atomic formulas $f_{j} \propto_{j} 0$ via (6.6). For this, we need to check that

$$
3 d_{H}\left(\mathcal{X}_{\ell}(f, \Phi), \mathrm{S}(f, \Phi)\right)<\varepsilon<\frac{1}{14 D^{3 / 2} \bar{\kappa}(f)} .
$$

However, the right-hand inequality holds by assumption and the left-hand inequality follows from the Sampling Theorem 6.5 (it is immediate to check that $13 D^{2} \bar{\kappa}(f)^{2} r_{\ell}<1$ follows from our hypothesis).

We provide now the proof of the crucial Homology Witness Theorem 2.4.

Proof of Theorem 2.4. Without loss of generality, we can assume that $\Phi$ is in DNF, i.e., it is of the form $\bigvee_{i \in I} \phi_{i}$ with each $\phi_{i}$ purely conjunctive. We can further assume that no polynomial appears twice in any of the $\phi_{i}$. We can do these assumptions because they change neither the semialgebraic set $\mathbf{S}(f, \Phi)$ nor the simplicial complex $\mathfrak{C}$ defined in (6.8). 
We will use the Inclusion-Exclusion Transfer (Corollary 5.5) to show that both $\mathrm{S}(f, \Phi)$ and $\mathfrak{C}$ have the same homology as the algebraic neighborhood $\mathrm{S}_{\rho}^{\circ}(f, \Phi)$ for $\rho=6 D^{1 / 2} \varepsilon$. Note that for this $\rho$ we have

$$
\sqrt{2} \bar{\kappa}(f) \rho=\sqrt{2} \bar{\kappa}(f) 6 D^{1 / 2} \varepsilon \leq \frac{\sqrt{2} \bar{\kappa}(f) 6 D^{1 / 2}}{14 D^{3 / 2} \bar{\kappa}(f)}<1 .
$$

We can then use Theorem 2.7 to deduce that, for all $J \subseteq I$, the inclusion

$$
\bigcap_{j \in J} \mathrm{~S}\left(f, \phi_{j}\right)=\mathrm{S}\left(f, \wedge_{j \in J} \phi_{j}\right) \subseteq \mathrm{S}_{\rho}^{\circ}\left(f, \wedge_{j \in J} \phi_{j}\right)=\bigcap_{j \in J} \mathrm{~S}_{\rho}^{\circ}\left(f, \phi_{j}\right)
$$

induces an isomorphism in homology. In addition, we have

$$
\bigcup_{i \in I} \mathrm{~S}\left(f, \phi_{i}\right)=\mathrm{S}(f, \Phi) \text { and } \bigcup_{i \in I} \mathrm{~S}_{\rho}^{\circ}\left(f, \phi_{i}\right)=\mathrm{S}_{\rho}^{\circ}(f, \Phi)
$$

so we can apply the Inclusion-Exclusion Transfer to the families $\left\{\mathrm{S}\left(f, \phi_{i}\right)\right\}_{i \in I}$ and $\left\{\mathrm{S}_{\rho}^{\circ}\left(f, \phi_{i}\right)\right\}_{i \in I}$ to deduce that the inclusion

$$
\mathrm{S}(f, \Phi) \hookrightarrow \mathrm{S}_{\rho}^{\circ}(f, \Phi)
$$

induces an isomorphism in homology.

We now need to show that $\mathfrak{C}$ and $\mathrm{S}_{\rho}^{\circ}(f, \Phi)$ have the same homology. To do so, for $J \subseteq\{1, \ldots, q\}$ and $\propto \in\{\leq,=, \geq\}^{J}$, we define the closed set $\mathcal{X}_{J}^{\propto}:=\cap_{j \in J} \mathcal{X}_{j}^{\alpha_{j}}$. We also let $\psi_{J}^{\propto}:=\bigwedge_{j \in J}\left(f_{j} \propto_{j} 0\right)$. By construction, we have

$$
\mathrm{S}\left(f, \psi_{J}^{\propto}\right)=\bigcap_{j \in J} S_{j}^{\propto_{j}}
$$

where the $S_{j}^{\propto_{j}}$ are the $3 q$ atomic sets associated with $f \in \mathcal{H}_{\boldsymbol{d}}[q]$ defined in (2.3).

We first prove that for all $z$ in the Euclidean neighborhood $\mathcal{U}\left(\mathcal{X}_{J}^{\alpha}, \varepsilon\right)$, we have

$$
d_{\mathbb{S}}\left(\frac{z}{\|z\|}, \mathrm{S}\left(f, \psi_{J}^{\propto}\right)\right)<6 \varepsilon .
$$

Indeed, for all $y_{0}, y_{1} \in \mathbb{S}^{n}$,

$$
d_{\mathbb{S}}\left(y_{0}, y_{1}\right) \leq \frac{\pi}{2} d\left(y_{0}, y_{1}\right) \leq 2 d\left(y_{0}, y_{1}\right) .
$$

Consequently,

$$
\begin{aligned}
d_{\mathbb{S}}\left(\frac{z}{\|z\|}, \mathrm{S}\left(f, \psi_{J}^{\propto}\right)\right) & \leq 2 d\left(\frac{z}{\|z\|}, \mathrm{S}\left(f, \psi_{J}^{\propto}\right)\right) \\
& <2 d\left(\frac{z}{\|z\|}, z\right)+2 d\left(z, \mathcal{X}_{J}^{\propto}\right)+2 d_{H}\left(\mathcal{X}_{J}^{\propto}, \mathrm{S}\left(f, \psi_{J}^{\propto}\right)\right) \\
& \leq 2 d\left(\frac{z}{\|z\|}, z\right)+2 d\left(z, \mathcal{X}_{J}^{\propto}\right)+2 \varepsilon \\
& =2 d\left(z, \mathbb{S}^{n}\right)+2 d\left(z, \mathcal{X}_{J}^{\propto}\right)+2 \varepsilon \\
& \leq 4 d\left(z, \mathcal{X}_{J}^{\propto}\right)+2 \varepsilon \\
& \leq 6 \varepsilon,
\end{aligned}
$$


where the second line follows from the triangular inequality for the Hausdorff distance, the third one from $d_{H}\left(\mathcal{X}_{J}^{\propto}, \mathrm{S}\left(f, \psi_{J}^{\propto}\right)\right)<\varepsilon / 3$, the fourth one from the fact that $\frac{z}{\|z\|}$ is the nearest point to $z$ in $\mathbb{S}^{n}$, the fifth one from $\mathcal{X}_{J}^{\alpha} \subseteq \mathbb{S}^{n}$ and the sixth and last one from $z \in \mathcal{U}\left(\mathcal{X}_{J}^{\alpha}, \varepsilon\right)$. Hence we have shown (6.10). As the set $\mathcal{U}\left(\mathcal{X}_{J}^{\alpha}, \varepsilon\right)$ is not included in the sphere $\mathbb{S}^{n}$ it will be convenient to consider, for any set $S \subseteq \mathbb{S}^{n}$ the cone

$$
\widehat{S}:=\{\lambda x \mid \lambda>0, x \in S\}
$$

over the spherical set $S$. Note that the inclusion

$$
S \stackrel{\simeq}{\hookrightarrow} \hat{S}
$$

is a homotopy equivalence since the map

$$
\widehat{S} \times[0,1] \rightarrow \widehat{S},(p, t) \mapsto \frac{p}{(1-t)+t\|p\|_{2}}
$$

induces a continuous retraction of $\widehat{S}$ onto $S$. These two spaces thus have the same homology. We will briefly write $\widehat{\mathcal{U}}$ and $\widehat{S}$ to denote the cone over the corresponding neighborhoods. As a consequence of (6.10) we deduce that

$$
\mathcal{U}\left(\mathcal{X}_{J}^{\propto}, \varepsilon\right) \subseteq \widehat{\mathcal{U}}_{\mathbb{S}}\left(\mathrm{S}\left(f, \psi_{J}^{\propto}\right), 6 \varepsilon\right) \subseteq \widehat{\mathrm{S}}_{\rho}^{\circ}\left(f, \psi_{J}^{\propto}\right)
$$

the last by (2.4) and the definition of $\rho$. We therefore have the inclusions

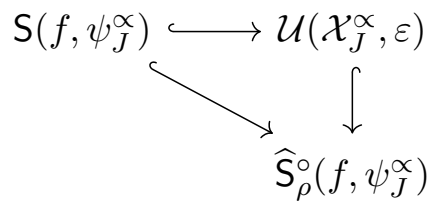

the horizontal arrow by hypothesis and the diagonal by composition.

We now note that $\mathrm{S}\left(f, \psi_{J}^{\alpha}\right) \hookrightarrow \mathcal{U}\left(\mathcal{X}_{J}^{\alpha}, \varepsilon\right)$ induces an isomorphism of homology by Theorem 2.3 and that so does $\mathrm{S}\left(f, \psi_{J}^{\propto}\right) \hookrightarrow \widehat{\mathrm{S}}_{\rho}^{\circ}\left(f, \psi_{J}^{\propto}\right)$, now by Theorem 2.7 and (6.11). This implies that the inclusion $\mathcal{U}\left(\mathcal{X}_{J}^{\propto}, \varepsilon\right) \hookrightarrow \widehat{\mathrm{S}}_{\rho}^{\circ}\left(f, \psi_{J}^{\propto}\right)$ induces the isomorphism

$$
H_{*}\left(\mathcal{U}\left(\mathcal{X}_{J}^{\propto}, \varepsilon\right)\right) \simeq H_{*}\left(\widehat{S}_{\rho}^{\circ}\left(f, \psi_{J}^{\propto}\right)\right) .
$$

Thus, the map

$$
\pi: \check{\mathrm{C}}_{\varepsilon}\left(\psi_{J}^{\propto}\right) \rightarrow \mathcal{U}\left(\mathcal{X}_{J}^{\propto}, \varepsilon\right)
$$

defined in Theorem 5.2 composed with the vertical arrow in (6.12) yields a map

$$
\pi^{\prime}: \check{\mathrm{C}}_{\varepsilon}\left(\psi_{J}^{\propto}\right) \rightarrow \widehat{\mathrm{S}}_{\rho}^{\circ}\left(f, \psi_{J}^{\propto}\right)
$$

that induces an isomorphism in homology, by Theorem 5.2 and (6.13). As the $\psi_{J}^{\propto}$ cover all the purely conjunctive formulas, up to equivalence, we have shown that, for every purely conjunctive formula $\phi$, the map

$$
\pi: \check{\mathrm{C}}_{\varepsilon}(\phi) \rightarrow \widehat{\mathrm{S}}_{\rho}^{\circ}(f, \phi)
$$

from Theorem 5.2 is well-defined, i.e., the image is contained in the codomain, and induces an isomorphism in homology. 
We come back to the general case. Since

$$
\mathfrak{C}=\bigcup_{i \in I} \check{\mathrm{C}}_{\varepsilon}\left(\phi_{i}\right) \text { and } \widehat{\mathrm{S}}_{\rho}^{\circ}(f, \Phi)=\bigcup_{i \in I} \widehat{\mathrm{S}}_{\rho}^{\circ}\left(f, \phi_{i}\right),
$$

the map

$$
\pi: \mathfrak{C} \rightarrow \widehat{\mathrm{S}}_{\rho}^{\circ}(f, \Phi)
$$

coming from Theorem 5.2 is well-defined, as we can guarantee that the image is contained in the codomain by the previous paragraph. This map induces an isomorphism in homology, by the Inclusion-Exclusion Transfer (Corollary 5.5) applied to the families $\left\{\check{\mathrm{C}}_{\varepsilon}\left(\phi_{i}\right)\right\}_{i \in I}$ and $\left\{\widehat{\mathrm{S}}_{\rho}^{\circ}\left(f, \phi_{i}\right)\right\}_{i \in I}$. This is so because, as we have just seen, the map $\pi$ induces an isomorphism in homology for purely conjunctive formulas, together with the equalities

$$
\bigcap_{j \in J} \check{\mathrm{C}}_{\varepsilon}\left(\phi_{j}\right)=\check{\mathrm{C}}_{\varepsilon}\left(\wedge_{j \in J} \phi_{j}\right) \text { and } \bigcap_{j \in J} \widehat{\mathrm{S}}_{\rho}^{\circ}\left(f, \phi_{j}\right)=\widehat{\mathrm{S}}_{\rho}^{\circ}\left(f, \wedge_{j \in J} \phi_{j}\right)
$$

for all $J \subseteq I$. Using (6.11) again we conclude that $\mathfrak{C}$ and $\boldsymbol{S}_{\rho}^{\circ}(f, \Phi)$ have the same homology.

We can conclude as we have shown that both $\mathbf{S}(f, \Phi)$ and $\mathfrak{C}$ have the same homology as $\mathrm{S}_{\rho}^{\circ}(f, \Phi)$ for the chosen $\rho$.

As a consequence of Proposition 6.6, we may construct the desired simplicial complex $\mathfrak{C}$ from the complexes $\breve{\mathrm{C}}_{\varepsilon}\left(\mathcal{X}_{j}^{\propto_{j}}\right)$ using the Boolean combination that yields $\mathrm{S}(f, \Phi)$ from the atoms $S_{j}^{\propto_{j}}$.

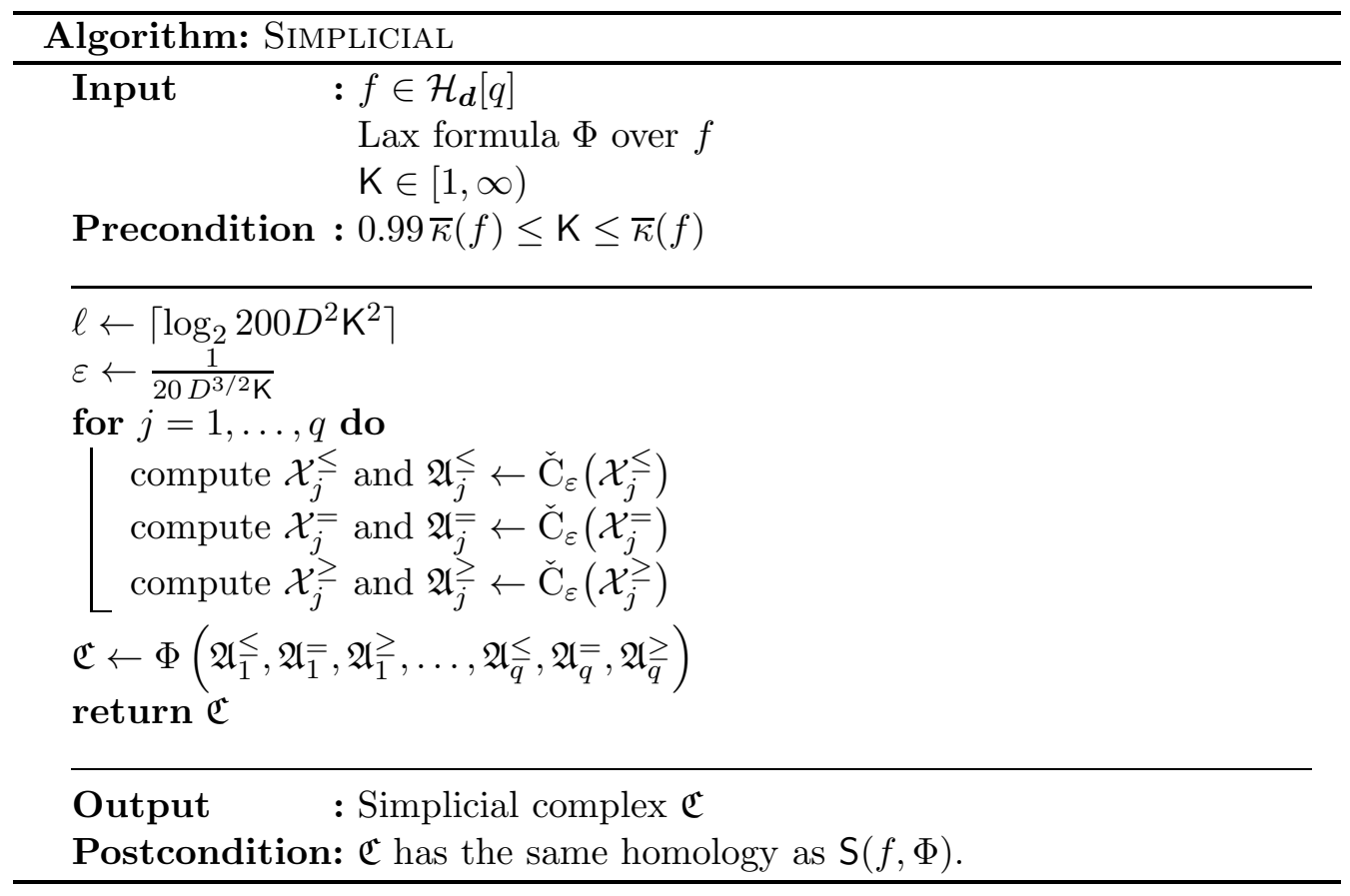

Proposition 6.7. Algorithm Simplicial is correct. The cost of running it on input $(f, \Phi, \mathrm{K})$ is bounded by

$$
(q+\operatorname{size}(\Phi))(n D \bar{\kappa}(f))^{\mathcal{O}\left(n^{2}\right)} .
$$

The number of faces of $\mathfrak{C}$ is bounded by $(n D \bar{\kappa}(f))^{\mathcal{O}\left(n^{2}\right)}$. 
Proof. It is straightforward to verify that the values the algorithm sets for $\ell$ and $\varepsilon$, along with the precondition on $\mathrm{K}$, guarantee that

$$
9 D^{1 / 2} \bar{\kappa}(f) r_{\ell}<\varepsilon<\frac{1}{14 D^{3 / 2} \bar{\kappa}(f)} .
$$

Hence, the hypothesis of Proposition 6.6 are satisfied and the correctness of the algorithm follows.

We next focus on complexity. The cost of computing an atomic cloud $\mathcal{X}_{j}^{\alpha_{j}}$ is that of evaluating $f$ at a point in $\mathcal{G}_{\ell}$ times the number of points in $\mathcal{G}_{\ell}$. The latter is $\left(n 2^{\ell}\right)^{\mathcal{O}(n)}$ by (6.4) and the former is $\mathcal{O}(N)=(n D)^{\mathcal{O}(n)}$ by (6.5). Using that $\mathrm{K} \leq \bar{\kappa}(f)$ it follows that we can compute one such atomic cloud with $\operatorname{cost}(n D \bar{\kappa}(f))^{\mathcal{O}(n)}$. Multiplying by $3 q$ we obtain the cost of computing all of them.

The computation of the set $F_{k}$ of $k$-faces of the Čch complex $\check{\mathrm{C}}_{\varepsilon}\left(\mathcal{X}_{j}^{\propto_{j}}\right)$ takes time $\left|\mathcal{X}_{j}^{\alpha_{j}}\right|{ }^{k} k^{\mathcal{O}(n)}$ (see [29, Lemma 4.2]). As $\left|\mathcal{X}_{j}^{\alpha_{j}}\right| \leq\left|\mathcal{G}_{\ell}\right|=\left(n 2^{\ell}\right)^{\mathcal{O}(n)}$ by (6.1), the computation of the sets $F_{k}$ for $k=0, \ldots, n$ (we are not interested in $k>n$ as $\operatorname{dim} S_{j}^{\propto_{j}} \leq n$ ) has cost

$$
\sum_{k=0}^{n}\left(n 2^{\ell}\right)^{\mathcal{O}(k n)} k^{\mathcal{O}(n)} \leq\left(n 2^{\ell}\right)^{\mathcal{O}\left(n^{2}\right)} \leq(n D \bar{\kappa}(f))^{\mathcal{O}\left(n^{2}\right)}
$$

Multiplying by $q$ we obtain the cost of computing all the $\check{\mathrm{C}}_{\varepsilon}\left(\mathcal{X}_{j}^{\propto_{j}}\right)$.

To compute the simplicial complex $\mathfrak{C}$ we compute, size $(\Phi)-1$ many times, a union or an intersection of two (already computed) Čech complexes $\mathfrak{C}_{1}$ and $\mathfrak{C}_{2}$ (see $\S 2.4$ ). The cost of each of these computations is linear in the size of $\mathfrak{C}_{1}$ and $\mathfrak{C}_{2}$ and hence, this final step has cost bounded by $\operatorname{size}(\Phi)(n D \bar{\kappa}(f))^{\mathcal{O}\left(n^{2}\right)}$. The statement now follows.

\subsection{Computation of homology groups}

The final procedure to obtain the homology of $\mathbf{S}(f, \Phi)$ computes the homology of the simplicial complex $\mathfrak{C}$ returned by Simplicial. The matrices $M_{k}$ corresponding to the boundary maps $\delta_{k}: C_{k} \rightarrow C_{k-1}$ for $k=1, \ldots, n$, where $C_{k}$ is the free Abelian group generated by the $k$-faces, have entries in $\{-1,0,1\}$. The Betti numbers $\beta_{0}(\mathfrak{C}), \ldots, \beta_{n-1}(\mathfrak{C})$, as well as the torsion coefficients, of $\mathfrak{C}$ are computed from these matrices via the computation of their Smith normal form. A description of how this is done is in [29, Proposition 4.3] where the following cost bound is also proved.

Proposition 6.8. The homology groups $H_{0}(\mathfrak{C}), \ldots, H_{n-1}(\mathfrak{C})$ of $\mathfrak{C}$ are computed from the matrices $M_{k}$ with a cost bounded by $n F^{\mathcal{O}(k)}$, where $F$ is the maximum over $k$ of the number of $k$-faces of $\mathfrak{C}$. In the case where $\mathfrak{C}$ is the simplicial complex returned by Simplicial with input $(f, \Phi, \mathrm{K})$, this total cost is

$$
(n D \bar{\kappa}(f))^{\mathcal{O}\left(n^{2}\right)} \text {. }
$$

\section{Affine Condition, Random Data}

\section{and Proof of the Main Result}

\subsection{Affine intersection condition}

Proposition 2.1 shows that, for well-posed tuples $p \in \mathcal{P}_{\boldsymbol{d}}[q]$, homogeneization reduces the computation of homology groups of semialgebraic sets to the same computation for 
spherical semialgebraic sets. In what follows we deal with this last unproved result in our overview. We start by defining the condition number $\bar{\kappa}_{\text {aff }}(p)$. The following example shows that taking $\bar{\kappa}\left(p^{\mathrm{h}}\right)$ with $p^{\mathrm{h}}=\left(p_{1}^{\mathrm{h}}, \ldots, p_{q}^{\mathrm{h}}\right)$ is not good enough.

Example 7.1. Consider the parabola $Y-X^{2} \in \mathcal{P}_{(2)}[1]$, whose homogenization gives the homogeneous polynomial $Z Y-X^{2}$ for which we can easily check that $\bar{\kappa}\left(Z Y-X^{2}\right)<\infty$, as zero is a regular value of this polynomial on the sphere. However, arbitrarily small perturbations of $Y-X^{2}$ inside $\mathcal{P}_{(2)}$ [1], e.g., those of the form $Y+\varepsilon Y^{2}-X^{2}$, can turn our description into that of an ellipse or a hyperbola, each of them having a topology different from that of a parabola.

Example 7.1 shows that $\bar{\kappa}\left(p^{\mathrm{h}}\right)$ alone does not capture ill-posedness. We note, however that for all $c>0, \bar{\kappa}\left(Z Y-X^{2}, c Z\right)=\infty$ as the parabola and the hyperplane at infinity do not intersect transversally. Hence, a condition measure of the form $\bar{\kappa}\left(p^{\mathrm{h}}, c X_{0}\right)$ for some $c>0$ would be a good measure. We have chosen this constant $c$ to be the norm $\left\|p^{\mathrm{h}}\right\|$ in our definition of $\mathrm{H}$ (cf. $\S 2.2$ ). This choice makes possible to prove Theorem 7.3 below.

Definition 7.2. The affine intersection condition number of $p \in \mathcal{P}_{\boldsymbol{d}}[q]$ is defined as

$$
\bar{\kappa}_{\text {aff }}(p):=\bar{\kappa}(\mathrm{H}(p)) .
$$

The following result, extending Theorem 3.10, is an immediate consequence of [15, Proposition 4.16] and Remark 3.9.

Theorem 7.3. Let $\bar{\Sigma}_{\boldsymbol{d}}^{\text {aff }}[q]:=\mathrm{H}^{-1}\left(\bar{\Sigma}_{\boldsymbol{d}}[q]\right)$. For all $p \in \mathcal{P}_{\boldsymbol{d}}[q]$ we have

$$
\bar{\kappa}_{\text {aff }}(p) \leq 4 D \frac{\|p\|}{d\left(p, \bar{\Sigma}_{\boldsymbol{d}}^{\text {aff }}[q]\right)} .
$$

Proof of Proposition 2.1. Let $\Phi^{>}$denote the formula

$$
\Phi^{>}:=\Phi\left(p_{1}^{\mathrm{h}}, \ldots, p_{q}^{\mathrm{h}}\right) \wedge\left(\|p\| X_{0}>0\right) .
$$

It is enough to check that $\mathrm{S}\left(\mathrm{H}(p), \Phi^{\mathrm{h}}\right)$ and $\mathrm{S}\left(\mathrm{H}(p), \Phi^{>}\right)$are homotopically equivalent, since it is well-known that $W(p, \Phi)$ is homeomorphic to $\mathrm{S}\left(\mathrm{H}(p), \Phi^{>}\right)$. This will follow from showing that $\mathrm{S}\left(\mathrm{H}(p), \Phi^{=}\right)$is collared in $\mathrm{S}\left(\mathrm{H}(p), \Phi^{\mathrm{h}}\right)$ where

$$
\Phi^{=}:=\Phi\left(p_{1}^{\mathrm{h}}, \ldots, p_{q}^{\mathrm{h}}\right) \wedge\left(\|p\| X_{0}=0\right),
$$

because then, by [15, Lemma 4.13], $\mathrm{S}\left(\mathrm{H}(p), \Phi^{>}\right)=\mathrm{S}\left(\mathrm{H}(p), \Phi^{\mathrm{h}}\right) \backslash \mathrm{S}\left(\mathrm{H}(p), \Phi^{=}\right)$would be homotopically equivalent to $\mathrm{S}\left(\mathrm{H}(p), \Phi^{\mathrm{h}}\right)$. Recall that a subset $B$ of a topological space $X$ is said to be collared in $X$ if there is a homeomorphism $h:[0,1) \times B \rightarrow V$ onto an open neighborhood $V$ of $B$ in $X$ such that $h(0, b)=b$ for all $b \in B$. Because of Brown's Collaring Theorem [11, 24] it is enough to show that $\mathrm{S}\left(\mathrm{H}(p), \Phi^{=}\right)$is locally collared in $\mathrm{S}\left(\mathrm{H}(p), \Phi^{\mathrm{h}}\right)$, i.e., that for every $x \in \mathrm{S}\left(\mathrm{H}(p), \Phi^{=}\right)$there is an open neighborhood $O_{x}$ of $x$ such that $\mathrm{S}\left(\mathrm{H}(p), \Phi^{=}\right) \cap O_{x}$ is collared in $\mathrm{S}\left(\mathrm{H}(p), \Phi^{\mathrm{h}}\right) \cap O_{x}$.

Fix $x \in \mathrm{S}\left(\mathrm{H}(p), \Phi^{=}\right)$. As $\bar{\kappa}(\mathrm{H}(p))=\bar{\kappa}_{\text {aff }}(p)<\infty$ we can take $r>0$ such that $\sqrt{2} \bar{\kappa}(\mathrm{H}(p)) r<1$ and apply Lemma 4.9 which guarantees the existence of a neighborhood $O_{x}$ of $x$ in $\mathbb{S}^{n}$ and trivializing coordinates around $x$ with respect to $(\mathrm{H}(p), r)$ on that neighborhood. On these coordinates we obtain formulas for $\mathrm{S}\left(\mathrm{H}(p), \Phi^{=}\right) \cap O_{x}$ and $\mathrm{S}\left(\mathrm{H}(p), \Phi^{\mathrm{h}}\right) \cap O_{x}$ by substituting $p_{j}^{\mathrm{h}}$ by $U_{j},\|p\| X_{0}$ by $U_{0}$, and the atoms of those polynomials having constant sign on $O_{x}$ by true or false appropriately. After doing this, we obtain 
a formula $\Xi$ over $\left(U_{i}\right)_{i \in S \backslash 0}$, where $S$ is as in Lemma 4.9 , for which $\mathrm{S}\left(\mathrm{H}(p), \Phi^{=}\right) \cap O_{x}$ is described by

$$
\Xi \wedge\left(U_{0}=0\right)
$$

and $\mathrm{S}\left(\mathrm{H}(p), \Phi^{\mathrm{h}}\right) \cap O_{x}$ by

$$
\Xi \wedge\left(U_{0} \geq 0\right)
$$

From this, it follows that the map

$$
\begin{aligned}
h:[0,1) \times \mathrm{S}\left(\mathrm{H}(p), \Phi^{=}\right) \cap O_{x} & \rightarrow \mathrm{S}_{r}^{\circ}\left(\mathrm{H}(p), \Phi^{=}\right) \cap \mathrm{S}\left(\mathrm{H}(p), \Phi^{\mathrm{h}}\right) \cap O_{x} \\
(t,(z, u)) & \mapsto\left(z, u_{0}+r t,\left(u_{i}\right)_{i \in S \backslash 0}\right)
\end{aligned}
$$

is a homeomorphism of $[0,1) \times \mathrm{S}\left(\mathrm{H}(p), \Phi^{=}\right) \cap O_{x}$ with an open neighborhood of $\mathrm{S}\left(\mathrm{H}(p), \Phi^{=}\right) \cap$ $O_{x}$ inside $\mathrm{S}\left(\mathrm{H}(p), \Phi^{\mathrm{h}}\right) \cap O_{x}$ for $r$ sufficiently small, since altering $u_{0}$ does not affect whether $\Xi$, in which $U_{0}$ does not appear, is true or not. Hence, $\mathrm{S}\left(\mathrm{H}(p), \Phi^{=}\right) \cap O_{x}$ is collared in $\mathrm{S}\left(\mathrm{H}(p), \Phi^{\mathrm{h}}\right) \cap O_{x}$ and the proof is complete.

\subsection{Random tuples in $\mathcal{P}_{\boldsymbol{d}}[q]$ and $\mathcal{H}_{\boldsymbol{d}}[q]$}

To obtain weak complexity estimates we endow the unit sphere $\mathbb{S}\left(\mathcal{P}_{\boldsymbol{d}}[q]\right)$ with the uniform measure. We observe that, as $\bar{\kappa}_{\text {aff }}(p)=\bar{\kappa}_{\text {aff }}(\lambda p)$ for all $\lambda>0$, the probability tail for $\bar{\kappa}_{\text {aff }}(p)$ is the same no matter whether $p$ is taken from the uniform distribution on $\mathbb{S}\left(\mathcal{P}_{\boldsymbol{d}}[q]\right)$ or from the standard Gaussian distribution on $\mathcal{P}_{\boldsymbol{d}}[q]$ with respect to the Weyl monomial basis $\left\{\left(\begin{array}{c}d_{j} \\ \alpha\end{array}\right) X^{\alpha}\right\}_{|\alpha|=d_{j}}$ for each $p_{j}$.

For any of these two distributions and for a condition number of the form $\mathscr{C}(a)=\frac{\|a\|}{d(a, \Sigma}$ where $\Sigma \subseteq \mathbb{R}^{p+1}$ is an algebraic cone defined as the zero set of a homogeneous polynomial $h$, the main result in [16] (see also [14, Theorem 21.1] for the bound below) gives estimates on the tail of $\mathscr{C}$ in terms of the degree of $h$ and the dimension of the ambient space: for all $t \geq(2 \operatorname{deg}(h)+1) / p$,

$$
\underset{a \in \mathbb{R}^{p+1}}{\operatorname{Prob}}\left\{\frac{\|a\|}{d(a, \Sigma)} \geq t\right\}=\underset{a \in \mathbb{S}^{p}}{\operatorname{Prob}}\left\{\frac{1}{d(a, \Sigma)} \geq t\right\} \leq 11 \frac{p \operatorname{deg}(h)}{t} .
$$

This result was used in [29] and subsequently in [15] (in conjunction with Theorem 7.3) to obtain bounds for the tail of $\kappa(f)$ and $\kappa^{*}(f, g)$. Our proof of the next result will be consequently succinct.

Proposition 7.4. For all $t \geq \frac{n 2^{n+1}(q+1)^{n+1} D^{n}+1}{N-1}$,

$$
\underset{p \in \mathbb{S}^{N-1}}{\operatorname{Prob}}\left\{\bar{\kappa}_{\text {aff }}(p) \geq t\right\} \leq \frac{44 D^{n+1}(N-1) n(2(q+1))^{n}}{t} .
$$

Proof. The set $\bar{\Sigma}_{\boldsymbol{d}}^{\text {aff }}[q]$ is contained in the zero set of a polynomial in $N$ variables of degree bounded by $n 2^{n}(q+1)^{n+1} D^{n}$ by [15, Corollary 4.21] and Remark 3.9. We now use Theorem 7.3 and (7.1).

\subsection{Proof of the Main Result}

We begin by exhibiting the algorithm HomOLOGY. 


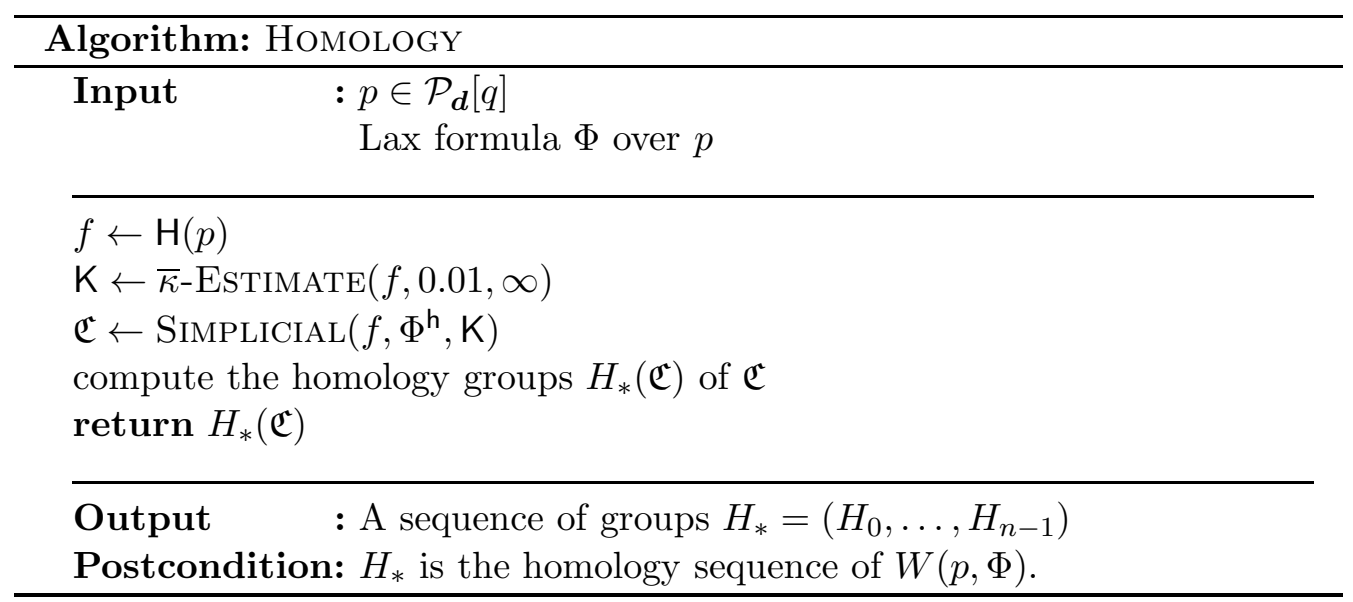

Its correctness is a trivial consequence of Propositions 2.1, 2.2 and 6.7. The last two, together with Proposition 6.8, yield the bound

$$
\begin{aligned}
\operatorname{cost}(p, \Phi) & \leq(q n D \bar{\kappa}(f))^{\mathcal{O}(n)}+(n D \bar{\kappa}(f))^{\mathcal{O}\left(n^{2}\right)}+(q+\operatorname{size}(\Phi))(n D \bar{\kappa}(f))^{\mathcal{O}\left(n^{2}\right)} \\
& \leq \operatorname{size}(\Phi) q^{\mathcal{O}(n)}(n D \bar{\kappa}(f))^{\mathcal{O}\left(n^{2}\right)}
\end{aligned}
$$

for the cost of the algorithm on input $(p, \Phi)$. This proves part (i) of Theorem 1.1.

For part (ii) we take $t=(n q D)^{c n}$ with $c>0$ large enough so that the hypothesis of Proposition 7.4 holds. Then, that proposition guarantees that

$$
\operatorname{Prob}_{p \in \mathbb{S}^{N-1}}\left\{\bar{\kappa}_{\text {aff }}(p) \geq(n q D)^{c n}\right\} \leq \frac{44 D^{n+1}(N-1) n(2(q+1))^{n}}{(n q D)^{c n}} \leq(n q D)^{-n}
$$

the last as $N=(n D)^{\mathcal{O}(n)}$ by (6.5) and by choosing $c$ large enough. It follows that with probability at least $1-(n D)^{-n}$ we have $\bar{\kappa}_{\text {aff }}(p) \leq(n D)^{c n}$ and hence, by part (i), $\operatorname{cost}(p, \Phi) \leq \operatorname{size}(\Phi) q^{\mathcal{O}(n)}(n D)^{\mathcal{O}\left(n^{3}\right)}$.

Finally, to prove part (iii), we take $t=2^{c \operatorname{size}(p, \Phi)}$. It is easy to see that we can choose $c$ large enough so that the hypothesis of Proposition 7.4 holds. Again, that proposition then guarantees that

$$
\operatorname{Prob}_{p \in \mathbb{S}^{N-1}}\left\{\bar{\kappa}_{\text {aff }}(p) \geq 2^{c \operatorname{size}(p, \Phi)}\right\} \leq \frac{44 D^{n+1}(N-1) n(2(q+1))^{n}}{2^{c \operatorname{size}(p, \Phi)}} \leq 2^{-\operatorname{size}(p, \Phi)}
$$

the last inequality by choosing $c$ large enough. As before, it follows that with probability at least $1-2^{-\operatorname{size}(p, \Phi)}$ we have $\bar{\kappa}_{\text {aff }}(p) \leq 2^{c \operatorname{size}(p, \Phi)}$ and hence, by part (i),

$$
\operatorname{cost}(p, \Phi) \leq \operatorname{size}(\Phi) q^{\mathcal{O}(n)}(n D)^{\mathcal{O}\left(n^{2}\right)} 2^{c \operatorname{size}(p, \Phi) n^{2}} \leq 2^{\mathcal{O}\left(\operatorname{size}(p, \Phi)^{1+\frac{2}{D}}\right)}
$$

the last since $\operatorname{size}(p, \Phi) \geq N=\Omega\left(n^{D}\right)$.

\subsection{Parallel computations}

The next result does not attempt to exhibit precise bounds. It only sketches a proof of weak parallel polynomial time. 
Proposition 7.5. Algorithm Homology parallelizes well. That is, it can be executed with

$$
\operatorname{size}(\Phi)\left(n q D \bar{\kappa}_{\text {aff }}(p)\right)^{n^{\mathcal{O}(1)}}
$$

parallel processors with a parallel time bounded by

$$
\operatorname{depth}(\Phi)\left(n \log _{2}\left(n q D \bar{\kappa}_{\text {aff }}(p)\right)\right)^{\mathcal{O}(1)}
$$

where depth $(\Phi)$ is the smallest depth of a tree with nodes $\vee$ and $\wedge$ evaluating $\Phi$.

If $p$ is drawn from the uniform distribution on $\mathbb{S}^{N-1}$, then the parallel cost with input $(p, \Phi)$ is bounded by $\operatorname{size}(p, \Phi)^{\mathcal{O}(1)}$ with probability at least $1-2^{-\operatorname{size}(p, \Phi)}$.

Proof. Each iteration of the repeat loop in $\bar{\kappa}$-Estimate can be fully parallelized. That is, the $(q n D \bar{\kappa}(f))^{\mathcal{O}(n)}$ evaluations done to compute $k$ are performed independently and then a maximum is taken with parallel cost $\mathcal{O}(n) \log _{2}(n q D \bar{\kappa}(f))$. As the loop is executed at most $\log _{2}(D \bar{\kappa}(f))+\mathcal{O}(1)$ times, the cost of $\bar{\kappa}$-Estimate is well within the claimed bounds.

The $3 q$ computations corresponding to the atomic sets in the for loop in Simplicial are done independently. For each of them, we first compute the cloud $\mathcal{X}_{j}^{\propto_{j}}$ and the the simplicial complex $\mathfrak{A}_{j}^{\alpha_{j}}=\check{\mathrm{C}}_{\varepsilon}\left(\mathcal{X}_{j}^{\alpha_{j}}\right)$. The computation of the cloud amounts to $(n D \bar{\kappa}(f))^{\mathcal{O}(n)}$ evaluations of $f$ at a point, which can be independently done. Each of them can be done in parallel time $\log _{2} N$. Again within the claimed bound.

The sets $F_{k}$ of $k$-faces of $\mathfrak{A}_{j}^{\alpha_{j}}$ can be computed independently for $j=1, \ldots, q$ and $k=0, \ldots, n$. It is well-known that these computations parallelize well (deciding whether a $k$-tuple of points is a $k$-face is deciding the truth of an existential formula, a problem whose parallel complexity is bounded in [4]). That is, we can compute each of them in time at most

$$
\left(n \log _{2}(n D \bar{\kappa}(f))\right)^{\mathcal{O}(1)} .
$$

We then compute $\mathfrak{C}$ in parallel time depth $(\Phi) \mathcal{O}\left(n^{2}\right) \log _{2}(n D \bar{\kappa}(f))$. The techniques used to, finally, compute $H_{*}(\mathfrak{C})$, basic linear algebra and the computation of the Smith normal form, parallelize well.

The last part of the statement is obtained as in the proof of Theorem 1.1(iii).

\section{References}

[1] D. Amelunxen and M. Lotz. Average-case complexity without the black swans. J. Complexity, 41:82-101, 2017.

[2] S. Basu. On bounding the Betti numbers and computing the Euler characteristic of semialgebraic sets. In Proceedings of the Twenty-Eighth Annual ACM Symposium on Theory of Computing, pages 408-417. ACM, 1996.

[3] S. Basu. Computing the first few Betti numbers of semi-algebraic sets in single exponential time. Journal of Symbolic Computation, 41(10):1125-1154, 2006.

[4] S. Basu, R. Pollack, and M.-F. Roy. On the combinatorial and algebraic complexity of quantifier elimination. Journal of the Assoc. Comput. Mach., 43:1002-1045, 1996.

[5] S. Basu, R. Pollack, and M.-F. Roy. Computing roadmaps of semi-algebraic sets on a variety. Journal of the Amer. Math. Soc., 33:55-82, 1999.

[6] C. Beltrán and L.M. Pardo. Smale's 17th problem: average polynomial time to compute affine and projective solutions. J. Amer. Math. Soc., 22(2):363-385, 2009. 
[7] R. Benedetti and J.-J. Risler. Real algebraic and semi-algebraic sets. Actualités Mathématiques. [Current Mathematical Topics]. Hermann, Paris, 1990.

[8] L. Blum, M. Shub, and S. Smale. On a theory of computation and complexity over the real numbers: NP-completeness, recursive functions and universal machines. Bull. Amer. Math. Soc. (N.S.), 21(1):1-46, 1989.

[9] J. Bochnak, M. Coste, and M.-F. Roy. Real algebraic geometry, volume 36 of Ergebnisse der Mathematik und ihrer Grenzgebiete (3). Springer-Verlag, Berlin, 1998. Translated from the 1987 French original, Revised by the authors.

[10] H. Bosse, M. Grötschel, and M. Henk. Polynomial inequalities representing polyhedra. Math. Program., 103(1, Ser. A):35-44, 2005.

[11] M. Brown. Locally flat imbeddings of topological manifolds. Annals of Mathematics, 75:331341, 1962.

[12] P. Bürgisser and F. Cucker. Exotic quantifiers, complexity classes, and complete problems. Found. Comput. Math., 9(2):135-170, 2009.

[13] P. Bürgisser and F. Cucker. On a problem posed by Steve Smale. Annals of Mathematics, 174:1785-1836, 2011.

[14] P. Bürgisser and F. Cucker. Condition, volume 349 of Grundlehren der mathematischen Wissenschaften. Springer-Verlag, Berlin, 2013.

[15] P. Bürgisser, F. Cucker, and P. Lairez. Computing the homology of basic semialgebraic sets in weak exponential time. J. ACM, 66(1):5:1-5:30, December 2018.

[16] P. Bürgisser, F. Cucker, and M. Lotz. The probability that a slightly perturbed numerical analysis problem is difficult. Mathematics of Computation, 77:1559-1583, 2008.

[17] J. Canny. The complexity of robot motion planning, volume 1987 of ACM Doctoral Dissertation Awards. MIT Press, Cambridge, MA, 1988.

[18] J. Canny. Computing roadmaps of general semi-algebraic sets. Comput. J., 36(5):504-514, 1993.

[19] J. Canny, D.Yu. Grigorev, and N.N. Vorobjov. Finding connected components of a semialgebraic set in subexponential time. Appl. Algebra Engrg. Comm. Comput., 2(4):217-238, 1992.

[20] F. Chazal, D. Cohen-Steiner, and A. Lieutier. A sampling theory for compact sets in Euclidean space. In Computational geometry (SCG'06), pages 319-326. ACM, New York, 2006.

[21] F. Chazal and A. Lieutier. The " $\lambda$-medial axis". Graphical Models, 67(4):304 - 331, 2005.

[22] F. Chazal and A. Lieutier. Weak feature size and persistant homology: computing homology of solids in $\mathbb{R}^{n}$ from noisy data samples. In Computational geometry (SCG'05), pages 255-262. ACM, New York, 2005.

[23] G.E. Collins. Quantifier elimination for real closed fields by cylindrical algebraic decomposition, pages 134-183. Lecture Notes in Comput. Sci., Vol. 33. Springer, Berlin, 1975.

[24] R. Connelly. A New Proof of Brown's Collaring Theorem. Proc. Am. Math. Soc., 27:180, 1971.

[25] F. Cucker. Approximate zeros and condition numbers. J. Complexity, 15:214-226, 1999.

[26] F. Cucker, T. Krick, G. Malajovich, and M. Wschebor. A numerical algorithm for zero counting. I: Complexity and accuracy. J. Complexity, 24:582-605, 2008.

[27] F. Cucker, T. Krick, G. Malajovich, and M. Wschebor. A numerical algorithm for zero counting. II: Distance to ill-posedness and smoothed analysis. J. Fixed Point Theory Appl., 6:285-294, 2009. 
[28] F. Cucker, T. Krick, G. Malajovich, and M. Wschebor. A numerical algorithm for zero counting. III: Randomization and condition. Adv. Applied Math., 48:215-248, 2012.

[29] F. Cucker, T. Krick, and M. Shub. Computing the homology of real projective sets. Found. Comput. Math., 18:929-970, 2018.

[30] F. Cucker and S. Smale. Complexity estimates depending on condition and round-off error. Journal of the Assoc. Comput. Mach., 46:113-184, 1999.

[31] J. Demmel. The probability that a numerical analysis problem is difficult. Math. Comp., 50:449-480, 1988.

[32] A.H. Durfee. Neighborhoods of algebraic sets. Trans. Amer. Math. Soc., 276(2):517-530, 1983.

[33] H. Edelsbrunner and J.L. Harer. Computational topology: An introduction. American Mathematical Society, Providence, RI, 2010.

[34] H. Federer. Curvature measures. Trans. Amer. Math. Soc., 93:418-491, 1959.

[35] C.G. Gibson, K. Wirthmüller, A.A. du Plessis, and E.J.N. Looijenga. Topological stability of smooth mappings. Lecture Notes in Mathematics, Vol. 552. Springer-Verlag, Berlin-New York, 1976.

[36] H.H. Goldstine and J. von Neumann. Numerical inverting matrices of high order, II. Proceedings of the Amer. Math. Soc., 2:188-202, 1951.

[37] D.Yu. Grigoriev. Complexity of deciding Tarski algebra. Journal of Symbolic Computation, 5:65-108, 1988.

[38] D.Yu. Grigoriev and N.N. Vorobjov. Solving systems of polynomial inequalities in subexponential time. Journal of Symbolic Computation, 5:37-64, 1988.

[39] D.Yu. Grigoriev and N.N. Vorobjov. Counting connected components of a semialgebraic set in subexponential time. Computational Complexity, 2:133-186, 1992.

[40] A. Hatcher. Algebraic topology. Cambridge University Press, Cambridge, 2002.

[41] J. Heintz, M.-F. Roy, and P. Solerno. Single exponential path finding in semi-algebraic sets II: The general case. In C.L. Bajaj, editor, Algebraic Geometry and its Applications, pages 449-465. Springer-Verlag, 1994.

[42] R.E. Hodel. An Introduction to Mathematical Logic. Dover Publications Inc., Mineola, New York, 2013.

[43] P. Koiran. The real dimension problem is $\mathrm{NP}_{\mathbb{R}}$-complete. J. Complexity, 15:227-238, 1999.

[44] E. Kostlan. Complexity theory of numerical linear algebra. J. of Computational and Applied Mathematics, 22:219-230, 1988.

[45] P. Lairez. A deterministic algorithm to compute approximate roots of polynomial systems in polynomial average time. Found. Comput. Math., 17(5):1265-1292, 2017.

[46] J. Mather. Notes on topological stability. Bull. Amer. Math. Soc. (N.S.), 49(4):475-506, 2012.

[47] J. von Neumann and H.H. Goldstine. Numerical inverting matrices of high order. Bull. Amer. Math. Soc., 53:1021-1099, 1947.

[48] P. Niyogi, S. Smale, and S. Weinberger. Finding the homology of submanifolds with high confidence from random samples. Discrete Comput. Geom., 39(1-3):419-441, 2008.

[49] J. Renegar. On the computational complexity and geometry of the first-order theory of the reals. Part I. Journal of Symbolic Computation, 13:255-299, 1992.

[50] D.J.S. Robinson. A course in the theory of groups, volume 80 of Graduate Texts in Mathematics. Springer-Verlag, New York, second edition, 1996. 
[51] J.J. Rotman. An introduction to algebraic topology, volume 119 of Graduate Texts in Mathematics. Springer-Verlag, New York, 1988.

[52] J.J. Rotman. An introduction to homological algebra. Universitext. Springer, New York, second edition, 2009.

[53] J.T. Schwartz and M. Sharir. A survey of motion planning and related geometric algorithms. Artificial Intelligence, 37:157-169, 1988.

[54] M. Shub and S. Smale. Complexity of Bézout's Theorem I: geometric aspects. Journal of the Amer. Math. Soc., 6:459-501, 1993.

[55] M. Shub and S. Smale. Complexity of Bézout's Theorem II: volumes and probabilities. In F. Eyssette and A. Galligo, editors, Computational Algebraic Geometry, volume 109 of Progress in Mathematics, pages 267-285. Birkhäuser, 1993.

[56] M. Shub and S. Smale. Complexity of Bézout's Theorem III: condition number and packing. Journal of Complexity, 9:4-14, 1993.

[57] M. Shub and S. Smale. Complexity of Bézout's Theorem V: polynomial time. Theoret. Comp. Sci., 133:141-164, 1994.

[58] M. Shub and S. Smale. Complexity of Bézout's Theorem IV: probability of success; extensions. SIAM J. of Numer. Anal., 33:128-148, 1996.

[59] S. Smale. Complexity theory and numerical analysis. In A. Iserles, editor, Acta Numerica, pages 523-551. Cambridge University Press, 1997.

[60] S. Smale. Mathematical problems for the next century. Mathematical Intelligencer, 20:7-15, 1998.

[61] E. Spanier. Algebraic Topology. McGraw-Hill, New York, 1966.

[62] A. Tarski. A decision method for elementary algebra and geometry. University of California Press, Berkeley and Los Angeles, Calif., 1951. 2nd ed.

[63] R. Thom. Ensembles et morphismes stratifiés. Bull. Amer. Math. Soc., 75:240-284, 1969.

[64] A.M. Turing. Rounding-off errors in matrix processes. Quart. J. Mech. Appl. Math., 1:287$308,1948$.

[65] H.R. Wüthrich. Ein Entscheidungsverfahren für die Theorie der reell-abgeschlossenen Körper. In E. Specker and V. Strassen, editors, Komplexität von Entscheidungsproblemen, volume 43 of Lect. Notes in Comp. Sci., pages 138-162. Springer-Verlag, 1976.

[66] G.M. Ziegler. Lectures on Polytopes. Graduate Texts in Mathematics. Springer New York, 2012.

\section{A On the smoothness assumption}

\section{in Thom's first isotopy lemma}

We begin observing that we can define Whitney stratifications of any subset of a manifold in the same manner we define Whitney stratifications of the manifold itself.

The following lemma will be instrumental in our proof.

Lemma A.1. Let $\mathcal{M}$ be a smooth manifold and $\mathcal{S}$ be a locally finite partition of a locally closed subset $\Omega \subset \mathcal{M}$. Then:

1. Let $\mathcal{S}^{c}$ be the partition whose elements are the connected components of the elements in $\mathcal{S}$. If $\mathcal{S}$ is a Whitney stratification, then so is $\mathcal{S}^{c}$. 
2. If $\mathcal{S}$ is a Whitney stratification with connected strata then it satisfies the boundary condition:

$$
\text { for } \sigma, \varsigma \in \mathcal{S} \text {, if } \varsigma \cap \bar{\sigma} \neq \varnothing \text {, then } \varsigma \subseteq \bar{\sigma} \text {. }
$$

3. Let $\mathcal{S}$ satisfy the boundary condition $(\mathrm{BC})$. Then $\mathcal{S}$ is a Whitney stratification if and only if for all $\sigma \in \mathcal{S}$,

$$
\mathcal{S}_{\mid \bar{\sigma}}:=\{\varsigma \in \mathcal{S} \mid \varsigma \subseteq \bar{\sigma}\}
$$

is a Whitney stratification of $\bar{\sigma}$.

4. Let $\mathcal{S}$ be a Whitney stratification, $\left\{\sigma_{i}\right\}_{i \in I} \subseteq \mathcal{S}$ a family of strata of the same dimension and $\mathcal{S}^{\prime}$ the partition obtained from $\mathcal{S}$ by replacing the $\sigma_{i}$ by its union. Then $\mathcal{S}^{\prime}$ is a Whitney stratification.

Proof. Parts 1 and 2 are [35, Ch. II, Theorem 5.6 and Corollary 5.7], respectively. For part 3, the fact that $\mathcal{S}$ satisfies (BC) implies that $\mathcal{S}_{\mid \bar{\sigma}}$ is a partition of $\bar{\sigma}$ whose elements are elements in $\mathcal{S}$. As a subset of a Whitney stratification is a Whitney stratification itself we have shown the 'only if' part. We next show the converse. For every $\sigma \in \mathcal{S}$, the fact that $\mathcal{S}_{\mid \bar{\sigma}}$ is a Whitney stratification implies that $\sigma \in \mathcal{S}_{\mid \bar{\sigma}}$ is a locally closed smooth submanifold. Next note that Whitney's condition b needs to be checked only for pairs $(\sigma, \varsigma) \in \mathcal{S}^{2}$ such that $\varsigma \cap \bar{\sigma} \neq \varnothing$. But the fact that $\mathcal{S}$ satisfies (BC) implies that, for any such pair, $\sigma, \varsigma \in \mathcal{S}_{\mid \bar{\sigma}}$ and therefore, it satisfies condition b because, by hypothesis, $\mathcal{S}_{\mid \bar{\sigma}}$ is a Whitney stratification.

We finally prove 4 . By the local character of Definition 4.1 of Whitney stratification, it is enough to check the conditions in this definition in some open neighborhood $U_{x}$ around each point $x \in \Omega$. Since $\mathcal{S}$ is locally finite, we can pick each $U_{x}$ such that $\mathcal{S}_{\mid U_{x}}$ is finite. Hence, without loss of generality, we can assume that $I$ is finite.

For all $i \neq j \in I$ we have $\sigma_{i} \cap \overline{\sigma_{j}}=\varnothing$. Otherwise, by [35, Ch. I, (1.1)], we would have $\operatorname{dim} \sigma_{i}<\operatorname{dim} \sigma_{j}$, contradicting our hypothesis. Hence, for all $i \in I$, there is an open set $U_{i}$ such that $\sigma_{i} \subseteq U_{i}$ and, for all $j \neq i, U_{i} \cap \overline{\sigma_{j}}=\varnothing$. It follows that $\cup \sigma_{i}$ is a locally closed smooth manifold. The verification of the conditions in Definition 4.1 for $\mathcal{S}^{\prime}$ is now straightforward.

To prove Theorem 4.4 we will rely on the following version of Thom's first isotopy lemma which is the one in [35, Ch. II, Theorem 5.2].

Theorem A.2. Let $\mathcal{M}$ be a smooth manifold and $\Omega \subseteq \mathcal{M}$ a locally closed subset with a Whitney stratification $\mathcal{S}$ and let $\alpha: \mathcal{M} \rightarrow \mathbb{R}^{k}$ be a smooth proper map such that:

- for each stratum $\sigma \in \mathcal{S}, \alpha_{\mid \sigma}: \sigma \rightarrow \mathbb{R}^{k}$ is surjective,

- for each stratum $\sigma \in \mathcal{S}, \alpha_{\mid \sigma}: \sigma \rightarrow \mathbb{R}^{k}$ is a smooth submersion.

Then $\alpha_{\mid X}$ is a trivial fiber bundle.

To deduce Theorem 4.4 from this result we will employ graphs of maps. This will allow us to transform our not necessarily smooth map into a smooth one, as it will be simply a projection.

Let $A$ and $B$ be smooth manifolds. Recall that the graph of a function $\varphi: A \rightarrow B$ is the set

$$
\Gamma_{\varphi}:=\{(a, b) \in A \times B \mid \varphi(a)=b\} .
$$


Associated with the graph we have the functions $i_{\varphi}: A \rightarrow \Gamma_{\varphi}$, given by $a \mapsto(a, \varphi(a))$, and $\pi: A \times B \rightarrow B$, given by $(a, b) \mapsto b$. Clearly, $\varphi=\pi \circ i_{\varphi}$. Also, it is easy to see, if $\varphi$ is a continuous map, then $\Gamma_{\varphi}$ is a closed subset of $A \times B$ and $i_{\varphi}$ is a homeomorphism between $A$ and $\Gamma_{\varphi}$. Finally, if $\varphi$ is a smooth map, then $\Gamma_{\varphi}$ is a closed smooth submanifold of $A \times B$ and $i_{\varphi}$ is a diffeomorphism between $A$ and $\Gamma_{\varphi}$. Given a subset $X \subseteq A$, we will consider

$$
\Gamma_{\varphi}(X):=\Gamma_{\varphi_{\mid X}}=\left\{(a, b) \in \Gamma_{\varphi} \mid a \in X\right\}=\{(a, \varphi(a)) \mid a \in X\} .
$$

It is again clear that if $\varphi$ is continuous and $X$ is a locally closed subset of $A$, then $\Gamma_{\varphi}(X)$ is a locally closed subset of $A \times B$. Moreover, if $\varphi$ is smooth and $X$ is a locally closed smooth submanifold of $A$, then $\Gamma_{\varphi}(X)$ is a locally closed smooth submanifold of $A \times B$.

Proof of Theorem 4.4. Consider the graph $\Gamma_{\alpha}$ of $\alpha$. Although not necessarily a manifold (as $\alpha$ may be non-smooth), it is a locally closed subset of $\mathcal{M} \times \mathbb{R}^{k}$. Next consider the partition of $\Gamma_{\alpha}$ given by

$$
\Gamma_{\alpha}(\mathcal{S}):=\left\{\Gamma_{\alpha}(\sigma) \mid \sigma \in \mathcal{S}\right\}
$$

and its associated partition $\Gamma_{\alpha}^{c}(\mathcal{S})$ as defined in Lemma A.1(1).

We claim that $\Gamma_{\alpha}^{c}(\mathcal{S})$ is a Whitney stratification of $\Gamma_{\alpha}$.

To prove the claim we first observe that $\Gamma_{\alpha}^{c}(\mathcal{S})=\Gamma_{\alpha}\left(\mathcal{S}^{c}\right)$. As, by Lemma A.1(1), $\mathcal{S}^{c}$ is a Whitney stratification and, by construction, has connected strata, Lemma A.1(2) shows that it satisfies the boundary condition $(\mathrm{BC})$. It follows that $\Gamma_{\alpha}^{c}(\mathcal{S})$ satisfies $(\mathrm{BC})$ as well.

Let $\sigma \in \mathcal{S}^{c}$ and $\sigma^{\prime} \in \mathcal{S}$ such that $\sigma \subseteq \sigma^{\prime}$. By the first hypothesis in our statement, there is an open neighborhood $U$ of $\overline{\sigma^{\prime}} \supseteq \bar{\sigma}$ and a smooth map $\varphi: U \rightarrow \mathbb{R}^{k}$ such that $\alpha_{\mid \sigma^{\prime}}=\varphi$. Clearly, $\alpha_{\mid \sigma}=\varphi$ as well. This implies that $\Gamma_{\alpha}\left(\mathcal{S}_{\mid \bar{\sigma}}^{c}\right)=\Gamma_{\varphi}\left(\mathcal{S}_{\mid \bar{\sigma}}^{c}\right)$ and $\Gamma_{\alpha}(\bar{\sigma})=\Gamma_{\varphi}(\bar{\sigma})$. Since $\varphi$ is smooth, $\Gamma_{\varphi}$ is a locally closed smooth submanifold and $i_{\varphi}: U \rightarrow \Gamma_{\varphi}$ is a diffeomorphism mapping the Whitney stratification $\mathcal{S}_{\mid \bar{\sigma}}^{c}$ to $\Gamma_{\alpha}^{c}\left(\mathcal{S}_{\mid \bar{\sigma}}\right)$ and the closed set $\bar{\sigma}$ to $\Gamma_{\alpha}(\bar{\sigma})$. Hence, by [35, Ch. I, (1.4)], $\Gamma_{\alpha}^{c}\left(\mathcal{S}_{\mid \bar{\sigma}}\right)$ is a Whitney stratification of $\Gamma_{\alpha}(\bar{\sigma})$.

As this happens for all strata $\Gamma_{\alpha}^{c}(\bar{\sigma})$ of the partition $\Gamma_{\alpha}^{c}(\mathcal{S})$ we may apply Lemma A.1(3) to deduce that $\Gamma_{\alpha}^{c}(\mathcal{S})$ is a Whitney stratification. We finally apply Lemma A.1(4) (several times for each dimension) to deduce that $\Gamma_{\alpha}(\mathcal{S})$ itself is a Whitney stratification. This proves the claim.

Since $\Gamma_{\alpha}(\mathcal{S})$ is a Whitney stratification of $\Gamma_{\alpha}$ the map $i_{\alpha}$ restricts to a diffeomorphism between $\sigma$ and $\Gamma_{\alpha}(\sigma)$, for all $\sigma \in \mathcal{S}$. In addition, as $\alpha=\pi \circ i_{\alpha}$, we have $\alpha_{\mid \sigma}=\pi_{\mid \Gamma_{\alpha}(\sigma)} \circ\left(i_{\alpha}\right)_{\mid \sigma}$ and, hence, as $\left(i_{\alpha}\right)_{\mid \sigma}$ is a diffeomorphism, $\pi_{\mid \Gamma_{\alpha}(\sigma)}$ is surjective if and only if $\alpha_{\mid \sigma}$ is so, and $\pi_{\mid \Gamma_{\alpha}(\sigma)}$ is a smooth submersion if and only if so is $\alpha_{\mid \sigma}$. In summary, the last two hypotheses of our statement imply the hypothesis of Theorem A.2, and consequently, that $\pi_{\mid \Gamma_{\alpha}}$ is a trivial bundle.

We can now conclude because a trivialization $h: \Gamma_{\alpha} \rightarrow F \times \mathbb{R}^{k}$ of $\pi_{\mid \Gamma_{\alpha}}$ induces the trivialization $h \circ i_{\alpha}$ of $\alpha: \mathcal{M} \rightarrow \mathbb{R}^{k}$. 